\title{
Molecular gas and stars in the translucent cloud MBM 18 (LDN 1569) ${ }^{\star \star \star \star, \star \star \star}$
}

\author{
J. Brand ${ }^{1}$, J. G. A. Wouterloot ${ }^{2}$, and L. Magnani ${ }^{3}$ \\ 1 INAF - Istituto di Radioastronomia \& Italian ALMA Regional Centre, via P. Gobetti 101, 40129 Bologna, Italy \\ e-mail: brand@ira.inaf.it \\ 2 Joint Astronomy Centre, 660 N. A'Ohoku Place, University Park, Hilo, HI 96720, USA \\ e-mail: j.wouterloot@jach.hawaii.edu \\ 3 Department of Physics and Astronomy, University of Georgia, Athens, GA 30602, USA \\ e-mail: loris@physast.athens.edu
}

Received 15 June 2012 / Accepted 5 August 2012

\section{ABSTRACT}

\begin{abstract}
Context. We investigate star formation in translucent, high-latitude clouds.
Aims. Our aim is to understand the star-formation history and rate in the solar neighbourhood.

Methods. We used spectroscopic observations of newly found candidate $\mathrm{H} \alpha$ emission-line stars to establish their pre-main-sequence nature. The environment was studied through molecular line observations of the cloud (MBM 18/LDN 1569) in which the stars are presumably embedded.

Results. Ten candidate $\mathrm{H} \alpha$ emission-line stars were found in an objective grism survey of a $\sim 1$ square degree region in MBM 18, of which seven have been observed spectroscopically in this study. Four of these have weak ( $|\mathrm{W}(\mathrm{H} \alpha)| \lesssim 5 \AA) \mathrm{H} \alpha$ emission, and six out of seven have spectral types M1-M4 V. One star is of type F7-G1 V, and has H $\alpha$ in absorption. The spectra of three of the M-stars may show an absorption line of LiI, although none of these is an unambiguous detection. The M-stars lie at distances between $\sim 60 \mathrm{pc}$ and $250 \mathrm{pc}$, while most distance determinations of MBM 18 found in the literature agree on 120-150 pc. For the six M-stars a good fit is obtained with pre-main-sequence isochrones indicating ages between 7.5 and $15 \mathrm{Myr}$. The mass of the molecular material, derived from the integrated ${ }^{12} \mathrm{CO}(1-0)$ emission, is $\sim 160 M_{\odot}$ (for a distance of $120 \mathrm{pc}$ ). This is much smaller than the virial mass $\left(\sim 10^{3} M_{\odot}\right)$, and the cloud is not gravitationally bound. Using a clump-finding routine, we identify 12 clumps from the CO-data, with masses between 2.2 and $22 M_{\odot}$. All clumps have a virial mass at least six times higher than their CO-mass, and thus none are in gravitational equilibrium. A similar situation is found from higher-resolution CO-observations of the northern part of the cloud.

Conclusions. Considering the relative weakness or absence of the $\mathrm{H} \alpha$ emission, the absence of other emission lines, and the lack of clear LiI absorption, the targets are not T Tauri stars. With ages between 7.5 and 15 Myr they are old enough to explain the lack of lithium in their spectra. Based on the derived distances, some of the stars may lie inside the molecular cloud. From the fact that the cloud as a whole, as well as the individual clumps, are not gravitationally bound, in combination with the ages of the stars we conclude that it is not likely that (these) stars were formed in MBM 18
\end{abstract}

Key words. stars: formation - stars: emission-line, Be - ISM: clouds - ISM: individual objects: MBM 18 (LDN 1569)

\section{Introduction}

Star formation in the Galaxy proceeds fitfully in both small and giant molecular clouds. The general mechanism (external or internal triggers leading to the formation of single stars or clusters) has been identified, though many details still must be worked out (see, e.g., Stahler \& Palla 2004). Among the lower-mass clouds, the star-formation capability of translucent clouds (objects with $1<A_{\mathrm{V}}<5 \mathrm{mag}$ ) has not been welldetermined yet. Some clouds near the translucent/dark cloud demarcation clearly form stars (e.g., MBM $12^{1}$, also known as

\footnotetext{
* Partly based on observations collected at the European Southern Observatory (ESO) using the ESO 3.6-m and the Swedish-ESO Submillimetre Telescope (SEST), La Silla, Chile.

$\star \star$ Appendix A is available in electronic form at http://www . aanda.org

$\star \star \star$ The full spectra are only available at the CDS via anonymous ftp to cdsarc.u-strasbg.fr $(130.79 .128 .5)$ or via http://cdsarc.u-strasbg.fr/viz-bin/qcat?]/A+A/547/A85

1 The MBM-identifier comes from the original catalogue of Magnani, Blitz \& Mundy (1985)
}

LDN 1453/4/7/8; Luhman 2001), while other, somewhat similar objects, do not (MBM 40; Magnani et al. 1996a). The reasons for this marked difference in star-forming capability are not clear. Unfortunately, the samples of star-forming vs. nonstar-forming translucent clouds are so small that it is difficult to recognize patterns or trends. While more than a hundred translucent clouds are known to exist (the vast majority identified at high Galactic latitudes - see Magnani et al. 1996b), less than a dozen have been thoroughly searched for evidence of star formation. Of these, only MBM 12 and MBM 20 (LDN 1642) show unambiguous evidence of low-mass star formation. About a halfdozen other clouds have some indication of possible star formation; see review by McGehee (2008). The question of whether or not stars form in translucent clouds is important because of the transient nature of these objects. Most translucent clouds at high Galactic latitudes are not gravitationally bound and are breaking up on timescales of $10^{6}$ years (Magnani et al. 1985). While a given translucent cloud may be gravitationally unbound, individual clumps within the cloud can be bound and potentially host star formation. Thus, the mechanism by which low-mass star formation begins in these objects is likely to differ from 


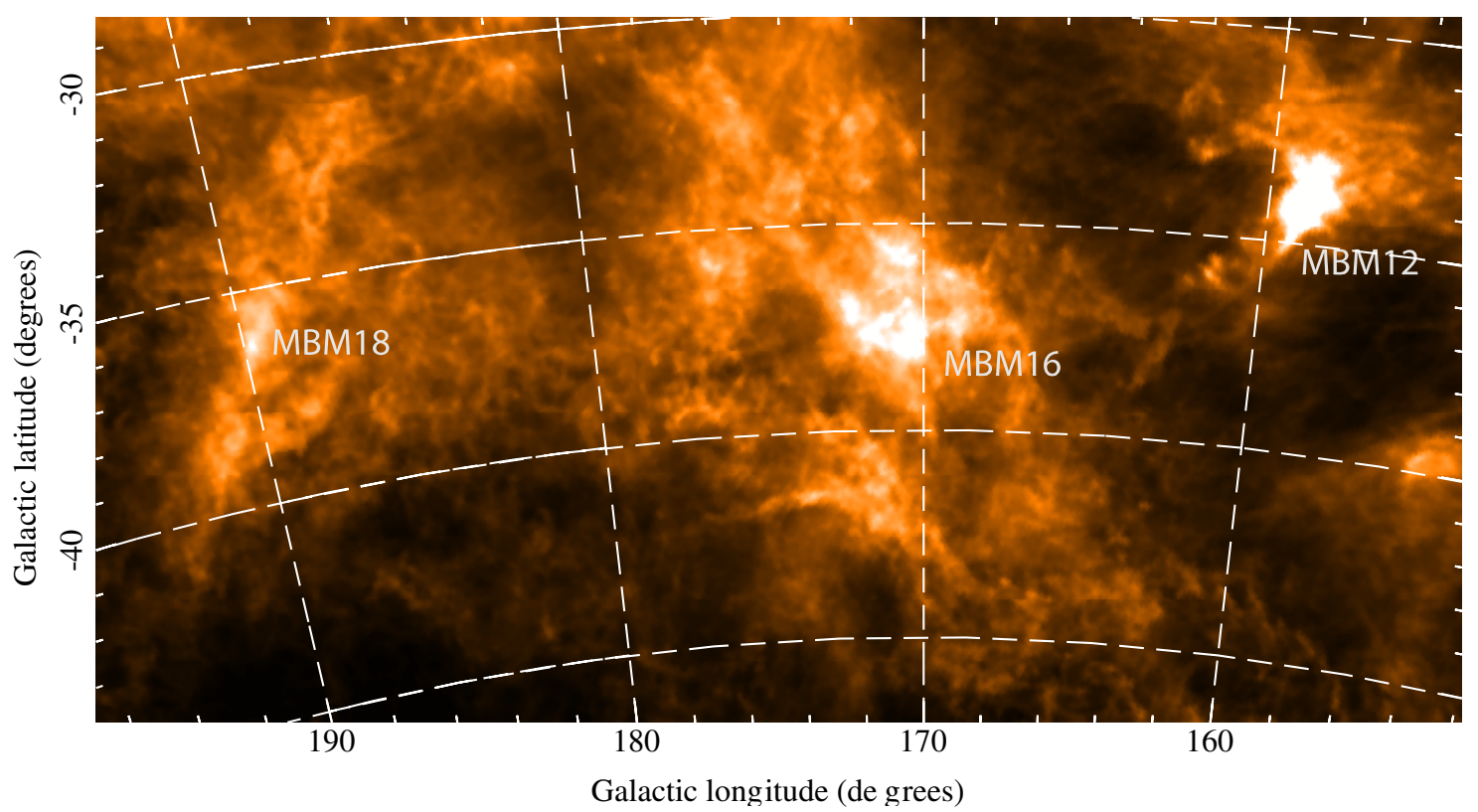

Fig. 1. Map of the colour excess $E(B-V)$ from Schlegel et al. 1998. The map is centred at $l \approx 174^{\circ}, b=-37^{\circ}$ and covers roughly $40^{\circ} \times 15^{\circ}$; a grid in galactic coordinates has been superposed. The minimum and maximum values in the map are 0.03 and 2.09 mag, respectively. The 3 principal features in this map from left to right are LDN 1569 (MBM 18), MBM 16 slightly to the right of the centre in the map, and MBM 12 (LDN 1453/4/7/8). MBM 12 is a known star-forming region with a young T-association that includes about a dozen objects, MBM 16 is not known to be forming stars, and LDN 1569 (MBM 18) contains the possible pre-main-sequence stars discussed in the text.

the mechanisms invoked for the larger, denser, dark molecular clouds. Determining which translucent high-latitude clouds do form stars and which do not could potentially unravel the nature of this process and shed more light on star formation in all lowmass molecular clouds. To accomplish this objective, it is imperative to expand the list of translucent clouds that have been thoroughly searched for low-mass star formation.

A distinguishing characteristic of low-mass pre-mainsequence (T Tauri) stars is the presence of emission lines in their spectra, especially $\mathrm{H} \alpha$. Searches for T Tauri stars are often carried out through $\mathrm{H} \alpha$ objective grism surveys. Stars found in this way have to be studied spectroscopically, to confirm their premain-sequence nature (and to rule out, e.g., dMe or Be stars). We have carried out an objective prism search for $\mathrm{H} \alpha$ emission-line stars in various types of clouds with the ESO 3.6-m telescope (Sect. 2.1). One of our targets was the high-latitude translucent cloud LDN $1569\left(\mathrm{MBM} 18 ; b=-35^{\circ}\right)$. Located at a distance of $\sim 120-150 \mathrm{pc}$ (Penprase 1993; but see Sect. 4 ), $15^{\circ}$ southeast of the Taurus/Auriga dark cloud complex, the cloud is of similar size and structure to MBM 12, but has lower extinction by a factor of 3 (at a resolution of 6.'1; see Fig. 1). We have found ten candidate $\mathrm{H} \alpha$ emission-line stars in a $45^{\prime} \times 45^{\prime}$ area centred on this cloud.

To establish whether our candidates are T Tauri stars requires higher-resolution spectra to seek confirmation of the presence of $\mathrm{H} \alpha$ emission and to determine their spectral types. The presence of LiI absorption at $6708 \AA$ above a certain equivalent width threshold in the spectra (see e.g. Preibisch et al. 1998; Bertout 1989) would also give an indication of the stars' youth (cf. Luhman 2001). It also needs to be established whether the stars are associated with the cloud against which they are seen projected. Determining that the candidates from the objective grism survey are truly pre-main-sequence stars formed in MBM 18 would be an important result because it would extend the extinction range of those translucent clouds capable of forming stars down to objects with $\sim 2$ mag of visual extinction in their densest regions. There are currently no unambiguous signatures of star formation in any high-latitude cloud with $A_{\mathrm{V}}<4$ mag in its most opaque region. Moreover, the cloud is likely at $100-200 \mathrm{pc}$ from the Sun, thus the pre-main-sequence stars would be among the nearest such objects, and if the objects constitute a small association, this would be a significant finding, on a par with the discovery of the TW Hydrae association (e.g., de la Reza et al. 1989; Zuckerman et al. 2001, and references therein), and would reveal important information on the star-formation history and rate in the solar neighbourhood.

We also need to obtain information on the environment of the stars, in particular the dynamical state of the gas (gravitationally bound or not) of the cloud (and its substructures) towards which the stars are seen projected.

In this paper we present the results of higher-resolution spectroscopy of emission-line star candidates in MBM 18, and of molecular line observations of the gas in this cloud.

\section{Observations}

\subsection{ESO 3.6-m}

On 21 November 1982 we used the ESO 3.6-m telescope at La Silla, in combination with the triplet corrector and a $1540 \AA \mathrm{mm}^{-1}$ red-sensitive grism in its prime focus, to obtain a plate with low-resolution spectra of a 1 square degree area of LDN 1569, covering the wavelength range 6300-9000. . This range is determined by the use of a IV-N plate and an RG630 filter. Just prior to the observations the plate was sensitised in $\mathrm{AgNO}_{3}$, and later developed in D19. The field centre was $4^{\mathrm{h}} 02^{\mathrm{m}} 04.5^{\mathrm{s}}, 00^{\circ} 48^{\prime} 18^{\prime \prime}(\mathrm{J} 2000)$ (at offset -5 '. $1,-30$ ' 0 with respect to the $(0,0)$ reference position in the CO maps). The limiting red magnitude was about 21 . In the same run we obtained a similar plate of LDN 1641 in Orion (not a translucent cloud; Wouterloot \& Brand 1992; see their Fig. 1 for examples of the grism-spectra). 
Table 1. Coordinates and magnitudes of the candidate $\mathrm{H} \alpha$ emission-line stars, and log of TNG observations.

\begin{tabular}{|c|c|c|c|c|c|c|c|c|c|c|c|c|c|c|}
\hline \multirow[t]{2}{*}{ Star } & \multicolumn{6}{|c|}{ RA (J2000) Dec } & \multirow[t]{2}{*}{$F^{a}$} & \multirow[t]{2}{*}{$V^{a}$} & \multirow{2}{*}{$\begin{array}{c}J^{b} \\
\mathrm{mag}\end{array}$} & \multirow[t]{2}{*}{$H^{b}$} & \multirow[t]{2}{*}{$K^{b}$} & \multirow{2}{*}{$\begin{array}{c}\text { TNG } \\
\mathrm{dd} / \mathrm{mm} / \mathrm{yy}\end{array}$} & \multirow{2}{*}{$\begin{array}{l}\text { Int. time } \\
\mathrm{s}\end{array}$} & \multirow[t]{2}{*}{ Comment } \\
\hline & $\mathrm{h}$ & $\mathrm{m}$ & $\mathrm{s}$ & $\circ$ & ' & " & & & & & & & & \\
\hline$\overline{\mathrm{Ha} 1}$ & 04 & 00 & 42.17 & +00 & 45 & 09.9 & 14.90 & 15.69 & 12.10 & 11.50 & 11.26 & $14 / 11 / 10$ & $4 \times 1728$ & \\
\hline $\mathrm{Ha} 2$ & 04 & 00 & 43.13 & +00 & 53 & 17.4 & 15.06 & 15.96 & 11.92 & 11.27 & 11.03 & $15 / 11 / 10$ & $2 \times 1440$ & \\
\hline $\mathrm{Ha} 3$ & 04 & 00 & 55.40 & +01 & 04 & 40.0 & 16.98 & 17.62 & 14.56 & 13.72 & 13.47 & \multicolumn{2}{|c|}{ not observed } & \\
\hline $\mathrm{Ha} 4$ & 04 & 00 & 58.53 & +00 & 44 & 33.8 & 16.15 & 16.81 & 14.86 & 14.37 & 14.35 & $14 / 11 / 10$ & $4 \times 1728$ & in same slit as $\mathrm{Ha} 1$ \\
\hline $\mathrm{Ha} 5$ & 04 & 01 & 23.56 & +01 & 06 & 49.1 & 14.57 & 15.51 & 10.89 & 10.32 & 10.00 & $12 / 03 / 09$ & $4 \times 450$ & \\
\hline $\mathrm{Ha} 6$ & 04 & 01 & 24.70 & +01 & 07 & 22.8 & 16.50 & 17.86 & 11.89 & 11.29 & 10.94 & $12 / 03 / 09$ & $4 \times 450$ & in same slit as $\mathrm{Ha} 5$ \\
\hline $\mathrm{Ha} 7$ & 04 & 02 & 44.80 & +01 & 13 & 15.5 & 15.89 & 16.60 & 13.36 & 12.78 & 12.54 & $29 / 10 / 10$ & $4 \times 1620$ & \\
\hline $\mathrm{Ha} 8$ & 04 & 03 & 00.07 & +00 & 27 & 35.0 & 15.19 & 15.86 & 12.14 & 11.56 & 11.35 & $12 / 12 / 10$ & $2 \times 1620$ & \\
\hline $\mathrm{Ha} 9$ & 04 & 03 & 30.65 & +00 & 32 & 28.4 & 16.37 & 17.42 & 13.35 & 12.67 & 12.37 & \multicolumn{2}{|c|}{ not observed } & \\
\hline Ha10 & 04 & 03 & 35.75 & +00 & 34 & 13.0 & 17.88 & 18.74 & 13.63 & 13.09 & 12.75 & \multicolumn{2}{|c|}{ not observed } & \\
\hline
\end{tabular}

Notes. ${ }^{(a)}$ Photographic magnitude from the GSC; $F$-mag approximately corresponding to the Johnson $R$-mag. ${ }^{(b)}$ Infrared magnitudes from the 2MASS survey.

\section{2. $T N G$}

On 12 March 2009, on 29 October, 14 and 15 November, and on 12 December 2010 we used the low-resolution spectrograph (DOLORES) at the Italian Telescopio Nazionale Galileo (TNG) at La Palma (Spain) to perform long-slit spectroscopy on seven of the candidate $\mathrm{H} \alpha$ emission-line stars detected in the grismsurvey. The coordinates of the targets and their estimated magnitudes are listed in Table 1, which also presents the log of the TNG observations.

We used grism VHR-R, which covers a wavelength range of $6240-7720 \AA$ with a dispersion of $0.80 \AA /$ pix. The scale of the CCD detector is $0 .{ }^{\prime \prime} 252 /$ pixel. The observations were carried out with a slit width of $1^{\prime \prime}$ or $1 .{ }^{\prime \prime} 5$, depending on the seeing, resulting in a spectral resolution of $3.2 \AA$ and $4.8 \AA$, respectively.

To avoid problems with cosmic rays, several separate spectra per star were obtained. The number of spectra and the individual integration times are listed in Table 1. Two of the stars (Ha4 and Ha6) were observed simultaneously with another target (Ha1 and Ha5, respectively) by positioning the slit at an appropriate angle. The integration time was based on the brighter star in the slit, thus the signal-to-noise ratio for the other target is lower than for the primary one.

To allow absolute flux calibration the standard star Feige24 or Feige34 (for Ha5-Ha6) was observed immediately before or after the target observations, using the same instrumental setup as for the target observations. Flat-fielding was performed using 10 (5 for Ha5-Ha6) frames which were uniformly illuminated by a halogen lamp. Wavelength calibration was performed using an arc-spectrum of an $\mathrm{Ar}, \mathrm{Ne}+\mathrm{Hg}$, and $\mathrm{Kr}$ lamp, or a $\mathrm{Ne}+\mathrm{Hg}$ (for Ha7) comparison lamp. A bias frame, to be subtracted from the other frames before analysis, was constructed from ten individual bias frames. Flat-, arc-, and bias-frames were obtained on the same day as the science observations and with the same instrumental setup.

\subsection{Molecular line observations}

\subsubsection{KOSMA}

We mapped the complete LDN 1569 cloud in ${ }^{12} \mathrm{CO} J=$ 1-0 using the KOSMA 3-m telescope (Gornergrat, Zermatt, Switzerland) between September 12 and December 1, 1989. We observed 708 positions on a 4 arcmin raster using frequency switching. The frequency resolution of the acousto-optical spectral (AOS) backend used was $0.1665 \mathrm{MHz}\left(0.433 \mathrm{~km} \mathrm{~s}^{-1}\right)$. After folding the frequency-switched spectra, the resulting rms was $0.16-0.26 \mathrm{~K}\left(T_{\mathrm{A}}^{*}\right)$ with a median value of $0.21 \mathrm{~K}$. The full width at half maximum (FWHM) beam size at $115 \mathrm{GHz}$ was 3.'9, and the main-beam efficiency of the telescope was 0.78 .

\subsubsection{SEST}

The northern part of LDN 1569 was mapped with the $15-\mathrm{m}$ SEST (ESO, La Silla, Chile) in ${ }^{12} \mathrm{CO} J=1-0$ between November 18 and 26, 1988. We observed 1570 positions on a 40 or 80 arcsec raster using frequency switching. We used an AOS with a spectral resolution of $0.043 \mathrm{MHz}\left(0.113 \mathrm{~km} \mathrm{~s}^{-1}\right)$, and the rms in the spectra after folding was $0.06-0.40 \mathrm{~K}\left(T_{\mathrm{A}}^{*}\right)$ (median value $0.24 \mathrm{~K}$ ). The main-beam efficiency at $115 \mathrm{GHz}$ was 0.70 . The FWHM beamsize of the SEST at this frequency is about $46^{\prime \prime}$.

The central region of the northern cloud was then mapped (289 positions) in ${ }^{13} \mathrm{CO} J=1-0$ in the same way. The rms (velocity resolution $\left.0.118 \mathrm{~km} \mathrm{~s}^{-1}\right)$ was $0.03-0.13 \mathrm{~K}\left(T_{\mathrm{A}}^{*}\right.$; median $0.10 \mathrm{~K})$. A few positions were observed in $\mathrm{C}^{18} \mathrm{O} J=1-0$ and CS $J=2-1$, both with rms $0.04 \mathrm{~K}\left(T_{\mathrm{A}}^{*}\right)$.

In addition, in January 1990 we observed 54 positions in ${ }^{12} \mathrm{CO}(2-1)$, mostly located around offset $-80 ",-80$ " and along two lines through the edge of the cloud, while three positions (at offsets $0^{\prime \prime}, 0^{\prime \prime},-80^{\prime \prime},-80^{\prime \prime}$, and $-800^{\prime \prime},-400^{\prime \prime}$ ) were observed in ${ }^{13} \mathrm{CO}(2-1)$. The velocity resolution was $0.051 \mathrm{~km} \mathrm{~s}^{-1}$ and the main-beam efficiency was about 0.5 . The rms $\left(T_{\mathrm{A}}^{*}\right.$; spectra smoothed to $0.113 \mathrm{~km} \mathrm{~s}^{-1}$ ) was $0.15-0.40 \mathrm{~K}$ for ${ }^{12} \mathrm{CO}(2-1)$, and $\sim 0.10 \mathrm{~K}$ for ${ }^{13} \mathrm{CO}(2-1)$, respectively.

\subsubsection{JCMT}

To confirm some results of the KOSMA map (namely that there is only weak $\mathrm{CO}$ emission at a far-IR maximum) we observed four positions with the JCMT 15-m telescope (Mauna Kea, Hawaii, USA) in ${ }^{12} \mathrm{CO} J=2-1$. The observations were carried out on 28 October 2008 in bad but stable weather. The rms $\left(T_{\mathrm{A}}^{*}\right)$ in those spectra was $0.26-0.33 \mathrm{~K}$ (resolution $0.040 \mathrm{~km} \mathrm{~s}^{-1}$ ); a standard observation on CRL618 showed the expected intensity. The beam FHWM at this frequency is $20^{\prime \prime}$, and the main-beam efficiency was 0.75 . The pointing accuracy was about $2^{\prime \prime}$. 

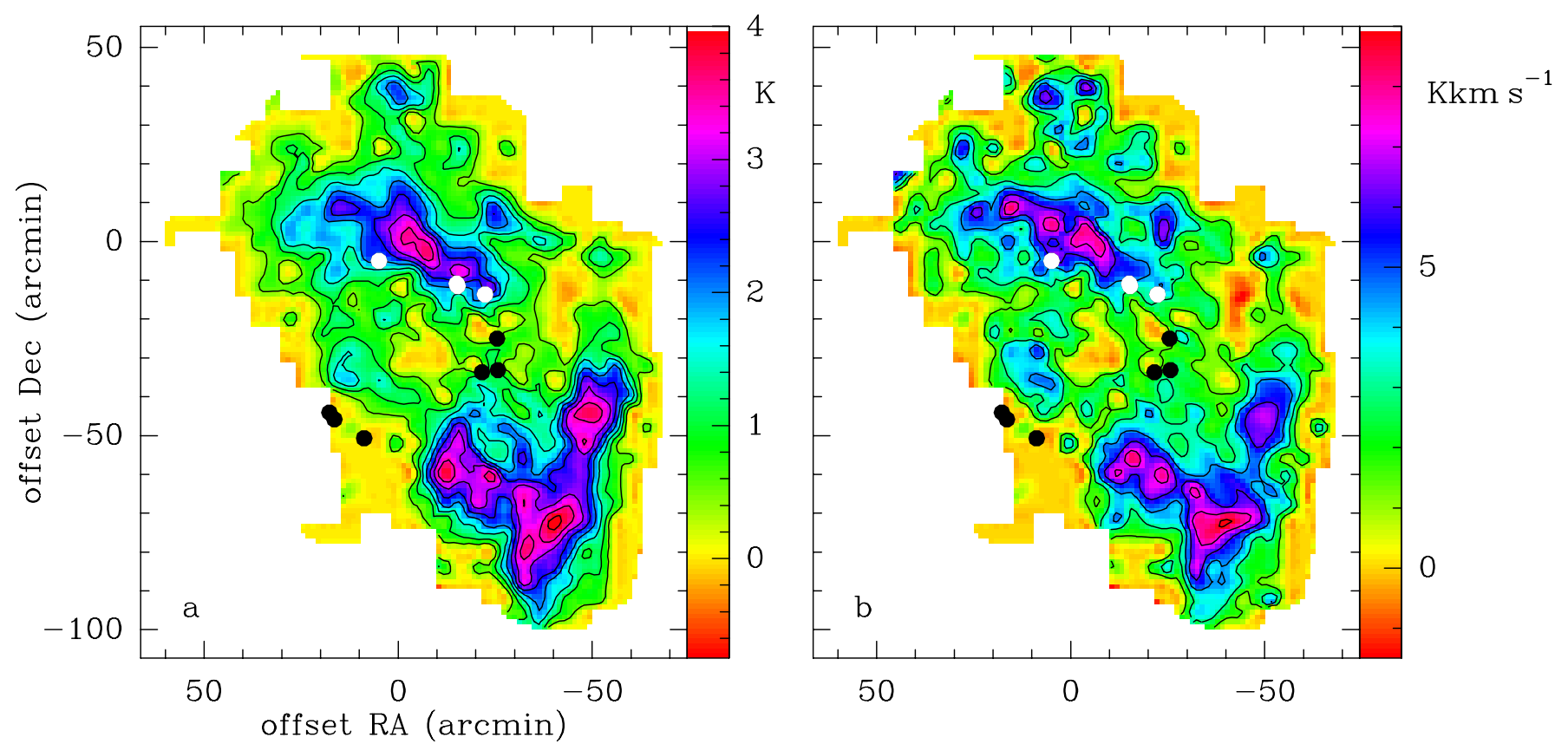

Fig. 2. a) Distribution of peak $T_{\mathrm{A}}^{*}$ of ${ }^{12} \mathrm{CO}(1-0)$ towards LDN 1569 obtained from KOSMA observations and Gaussian fits to the spectra. Contour levels are $0.5-4 \mathrm{~K}$ in steps of $0.5 \mathrm{~K}$. b) As a), but for the line-integrated emission $\int T_{\mathrm{A}}^{*} \mathrm{~d} v$. Contour levels are $1.5-9.0 \mathrm{~K} \mathrm{~km} \mathrm{~s}^{-1}$ in steps of $1.5 \mathrm{~K} \mathrm{~km} \mathrm{~s}^{-1}$. Offsets are in arcminutes relative to $4^{\mathrm{h}} 02^{\mathrm{m}} 25.0^{\mathrm{s}}, 01^{\circ} 18^{\prime} 17^{\prime \prime}$ (J2000). The filled circles indicate the positions of the candidate emissionline stars.

\subsubsection{Effelsberg}

Between March 28 and May 4, 1992 we used the 100-m Effelsberg telescope to observe $\mathrm{NH}_{3}(1,1)$ and $(2,2)$ towards the core of the SEST ${ }^{13} \mathrm{CO}$ cloud. We observed 26 positions on a 40 arcsec raster with a resolution of $12.177 \mathrm{kHz}\left(0.154 \mathrm{~km} \mathrm{~s}^{-1}\right)$. The rms of most spectra was $0.04-0.08 \mathrm{~K}\left(T_{\mathrm{mb}}\right)$. The beam FWHM at this frequency is $40^{\prime \prime}$.

\subsubsection{Medicina}

Between 10-12 October 2008, on 2 April and 16 May 2009, and on 19 January, 20 February, and 18 March 2011 we used the Medicina 32-m telescope ${ }^{2}$ to look for $\mathrm{H}_{2} \mathrm{O}\left(6_{16}-5_{23}\right)$ maser emission in the direction of all candidate $\mathrm{H} \alpha$ emission-line stars except stars $\mathrm{Ha} 3$ and $\mathrm{Ha} 4$.

We used a bandwidth of $10 \mathrm{MHz}$ and 1024 channels, resulting in a resolution of $9.77 \mathrm{kHz}\left(0.132 \mathrm{~km} \mathrm{~s}^{-1}\right)$; the half power beamwidth at $22.235 \mathrm{GHz}$ was $\sim 1$ '. 9 .

The telescope pointing model is typically updated a few times per year, and is quickly checked every few weeks by observing strong maser sources (e.g., W3 OH, Orion-KL, W49 N, Sgr B2, and W51). The pointing accuracy was always better than $25^{\prime \prime}$; the rms residuals from the pointing model were of the order of $8^{\prime \prime}-10^{\prime \prime}$.

Observations were taken in total power mode, with both $\mathrm{ON}$ and OFF scans of 5 min duration. The OFF position was taken $1.25 \mathrm{E}$ of the source position to rescan the same path as the $\mathrm{ON}$ scan. Typically, two ON/OFF pairs were taken at each position, though during the 01/2011 session we took ten pairs on Ha7.

The antenna gain as a function of elevation was determined by observing the continuum source DR 21 several times per day (for which we assume a flux density of $16.4 \mathrm{Jy}$ after scaling

\footnotetext{
2 The Medicina 32-m VLBI antenna is operated by INAF-Istituto di Radioastronomia.
}

the value of $17.04 \mathrm{Jy}$ given by Ott et al. 1994, for the ratio of the source size to the Medicina beam) at a range of elevations. Antenna temperatures were derived from total power measurements in position-switching mode. The integration time at each position was $10 \mathrm{~s}$ with $400 \mathrm{MHz}$ bandwidth. The zenith system temperature was about $90-100 \mathrm{~K}$ in clear weather conditions.

The daily gain curve was determined by fitting a polynomial curve to the DR 21 data; this was then used to convert antenna temperature to flux density for all spectra taken that day. From the dispersion of the single measurements around the curve, we found the typical calibration uncertainty to be $20 \%$.

\section{Data reduction and results}

\subsection{ESO 3.6-m}

The plate was scanned with the Astroscan measuring machine at the Leiden Observatory (NL) and digitized. Spectra of all stars on the plate were extracted and analysed with an algorithm that searches for a peak at the wavelength of $\mathrm{H} \alpha$ in the first-order spectrum. These spectra were also inspected visually. In this way we obtained a list of ten candidate $\mathrm{H} \alpha$ emission-line stars in a $45^{\prime} \times 45^{\prime}$ area in LDN 1569 (see Table 1). In Fig. 2 we show the locations of the stars superimposed on the map of integrated $\mathrm{CO}$ emission.

\section{2. $T N G$ - spectra}

Data were reduced with the IRAF package. From all science frames a bias was subtracted, after which they were divided by the normalised flat field. From each of the science frames the trace(s) of the star(s) were extracted and these were wavelength-calibrated using one of the frames with the arcspectrum. Each target was wavelength-calibrated with the arcspectrum extracted at the same location on the detector, to compensate for small deviations that might occur in the alignment 

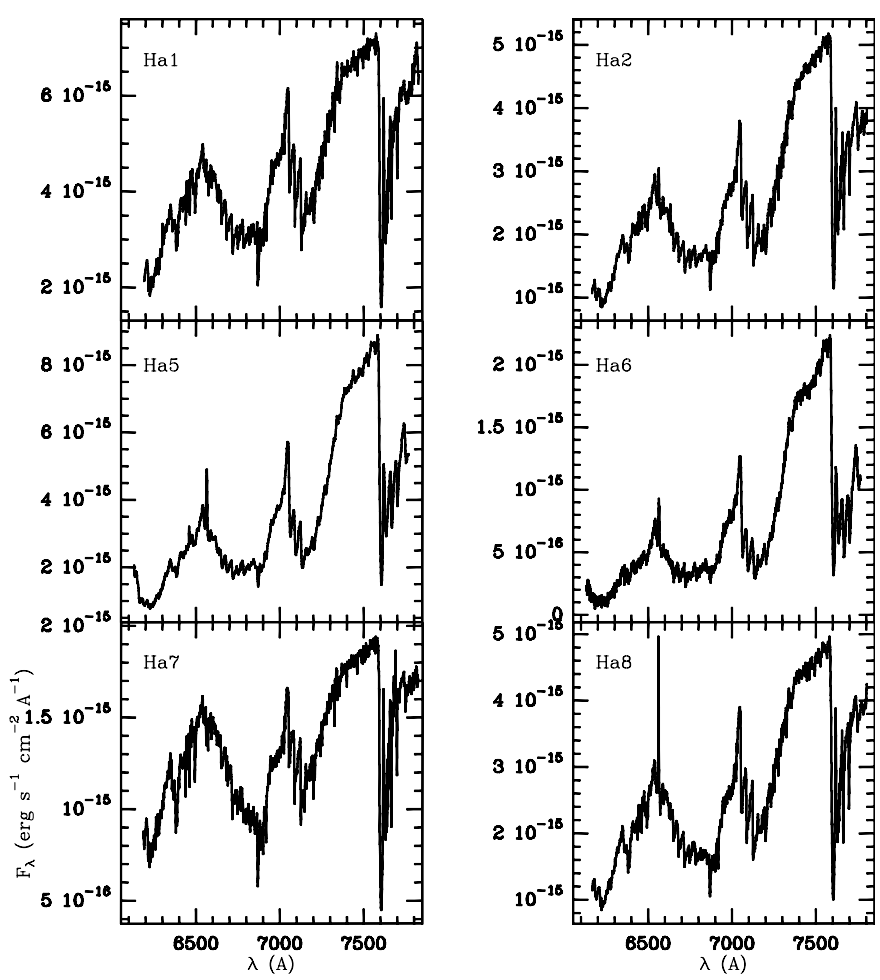

Fig. 3. Overview of the spectra obtained with Dolores at the TNG for the targets identified in each panel, showing the characteristic shape and absorption bands of M-type dwarf stars. More detailed spectra are shown in the appendix (on line version only).

of the reference emission lines across the detector. The spectra were then corrected for extinction, and flux-calibrated using the standard star observations. The individual one-dimensional wavelength- and flux-calibrated spectra of each target were then averaged into a final spectrum. To further correct the wavelength calibration, we used the sky lines that were subtracted from the stellar spectra. For each spectrum, Gaussian fits were made to tens of sky lines, and their wavelengths were compared to those listed in Osterbrock et al. (1996). Three stars were found to need a small correction: Ha2 (-1.5 $\AA$ ) and Ha5 and 6 (both $-2.2 \AA)$. For the other four stars the difference was negligible, although for the sky lines in Hal and Ha4 (which were observed in the same slit) the deviation between measured and literature wavelengths varied slightly, but systematically, with wavelengths between $6250 \AA$ and $7600 \AA$, while at longer wavelengths the deviations became rapidly larger (up to several Angstroms). The final spectra are shown in Fig. A.1 in the Appendix. Smaller versions (except for Ha4, see below) are presented in Fig. 3.

Following the criteria for the classification of late-type stars listed by Turnshek et al. (1985) and by comparison with the examples shown therein, we were able to roughly classify six of the stars as type M1 V - M4 V. Star Ha4 (see the full spectrum in Fig. A.2 in the Appendix) is clearly not a late-type dwarf star. Through visual comparison with spectra from the atlas of Jacoby et al. (1984) and with spectra from the SAO-FAST spectrograph ${ }^{3}$ we determine this star to be of type F7-G1 V.

Possibly Ha4 should not have been included in the sample. Looking at the grism-spectrum from the Astroscan measurement (Sect. 3.1), this star appears to have a much bluer spectrum than the others, confirming our DOLORES spectrum. The feature that

\footnotetext{
${ }^{3}$ http://tdc-www.harvard.edu/cgi-bin/arc/fsearch
}

we originally identified as $\mathrm{H} \alpha$ emission could have been a noise spike or be caused by dust on the grism-plate.

\subsection{KOSMA - CO}

All molecular line spectra were reduced using the CLASS reduction software, developed by the Observatoire de Grenoble and IRAM. It was necessary to subtract a higher order polynomial from some of the frequency-switched spectra, but because only narrow lines are present in the cloud, this did not affect the emission profiles.

Figure 2 shows the distribution of the ${ }^{12} \mathrm{CO}(1-0)$ emission of the whole cloud, observed with the KOSMA 3-m telescope. One can distinguish two main peaks in the northeast and southwest parts of the cloud, respectively. These peaks were already visible in the undersampled ${ }^{12} \mathrm{CO}(1-0)$ map from Magnani et al. (1985), and are seen to break up into a number of smaller clumps. The channel maps in Fig. 4 show that the northeastern peak has a velocity of about $8.5 \mathrm{~km} \mathrm{~s}^{-1}$, whereas the southwestern peak is at $10.5 \mathrm{~km} \mathrm{~s}^{-1}$. However, both peaks show internal velocity structure. The line width in the area with the strongest emission is about $1.8 \mathrm{~km} \mathrm{~s}^{-1}$. The total emission in the region observed with the KOSMA telescope (using $\int T_{\mathrm{mb}} \mathrm{d} v$ ) is $33999.2 \mathrm{~K} \mathrm{~km} \mathrm{~s}^{-1} \operatorname{arcmin}^{2}$, implying a CO-luminosity $L_{\mathrm{CO}}=41.4 \mathrm{~K} \mathrm{~km} \mathrm{~s}^{-1} \mathrm{pc}^{2}$ for a distance of $120 \mathrm{pc}$. Using the empirical relation $X=N\left(\mathrm{H}_{2}\right) / \int T_{\mathrm{mb}}(\mathrm{CO}) \mathrm{d} v=$ $1.8 \times 10^{20} \mathrm{~cm}^{-2}\left(\mathrm{~K} \mathrm{~km} \mathrm{~s}^{-1}\right)^{-1}$ (Dame et al. 2001), we find a mass $M_{\mathrm{CO}}=3.9 L_{\mathrm{CO}}=162 M_{\odot}$, which includes a factor of 1.36 to account for helium.

The data shown here represent the first fully sampled map of this translucent cloud, whose basic morphology was first delineated by Magnani et al. (1985). However, a perusal of this particular cloud using the Schlegel et al. (1998) dust map (Fig. 1) shows an extension to the west and south of the cloud. This is seen more clearly in Fig. 5, which shows the IRAS $100 \mu$ m map (or rather the improved reprocessed version of it: "IRIS"; from JPL-IPAC ${ }^{4}$ ), where we also indicate the KOSMA CO-contours, the location of the ESO 3.6-m grism-field (Sect. 2.1), and the position of the $\mathrm{H} \alpha$ candidate emission-line stars. It is not known whether the $100 \mu \mathrm{m}$ emission complexes to the west and south are also associated with $\mathrm{CO}$ emission. The southern high-latitude CO survey on a 1-degree sampling grid by Magnani et al. (2000) shows CO emission from only two positions in this essentially unmapped region. Although the dust emission from the southwestern clouds was not mapped in $\mathrm{CO}$, as can be seen from Fig. 1, the dust column density (and, thus, the gas column density) is significantly lower than in MBM 18. In any case, our spectra show that in our map of MBM 18 we have reached the edges of that cloud, and an eventual molecular cloud associated with the other dust emission complexes will be separate (though likely related) entities.

\section{4. $S E S T-C O$}

Figure 6 shows the distribution of the ${ }^{12} \mathrm{CO}(1-0)$ emission of the northeastern peak, observed with the SEST. Also here we observe a high degree of clumpiness. The angular resolution of the SEST at $115 \mathrm{GHz}$ corresponds to $0.027 \mathrm{pc}$ at a distance of $120 \mathrm{pc}$. Figure 7 shows that the central area has a velocity of about $8 \mathrm{~km} \mathrm{~s}^{-1}$, whereas the eastern and western parts are at a higher velocity. The line width in the area of the ${ }^{12} \mathrm{CO}$ peaks

${ }^{4}$ http://irsa.ipac.caltech.edu/data/IRIS 


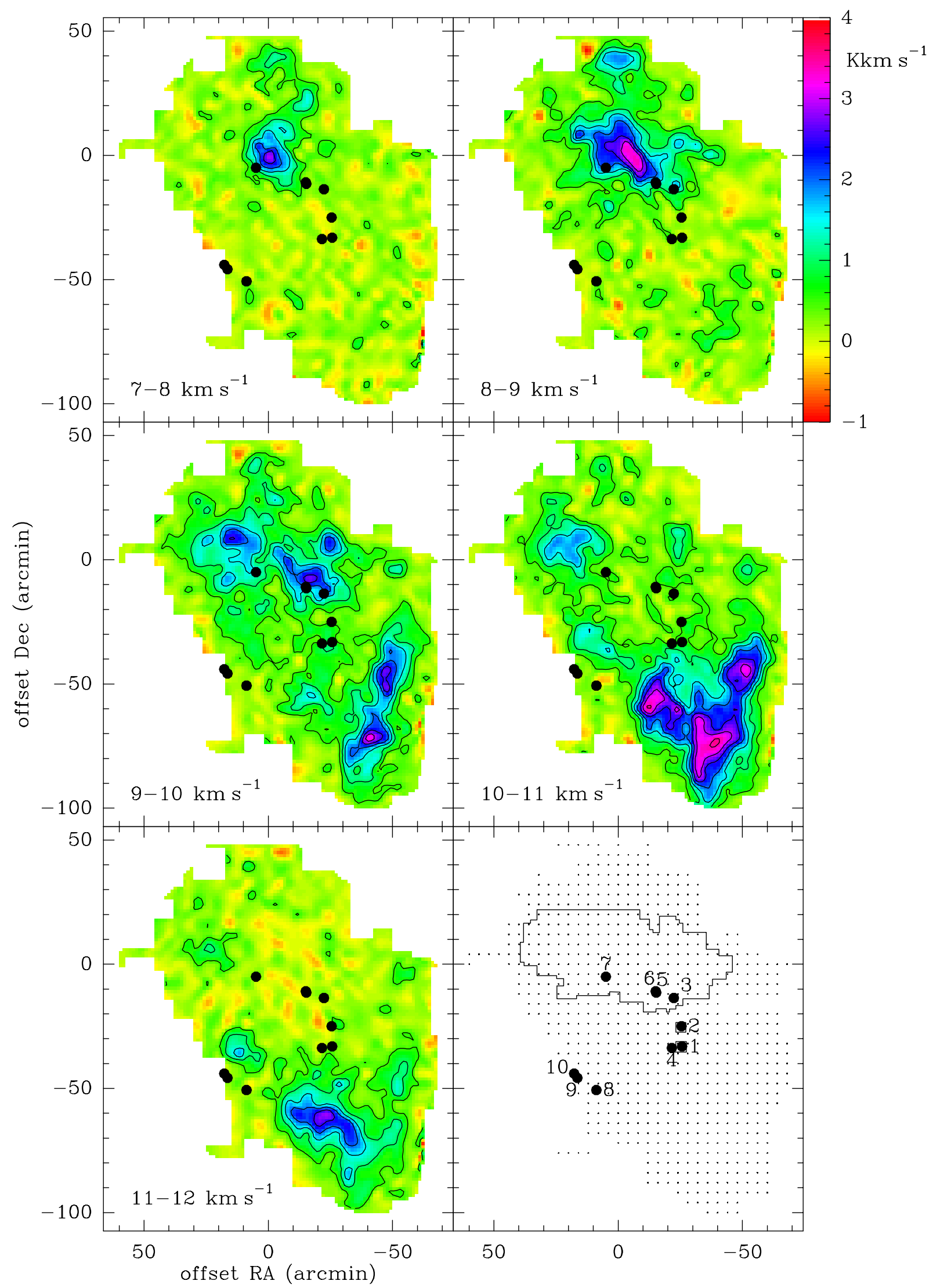

Fig. 4. KOSMA observations. ${ }^{12} \mathrm{CO}(1-0)$ line emission $\int T_{\mathrm{A}}^{*} \mathrm{~d} v$, integrated in velocity intervals of $1 \mathrm{~km} \mathrm{~s}^{-1}$ between 7 and $12 \mathrm{~km} \mathrm{~s}{ }^{-1}$. The lowest contour level and step are $0.5 \mathrm{~K} \mathrm{~km} \mathrm{~s}^{-1}$. The observed positions are indicated in the lower right panel. This panel also indicates the area observed with the SEST. The filled circles indicate the positions of the candidate emission-line stars, which are labeled with their numbers as in Table 1. Offsets as in Fig. 2. 


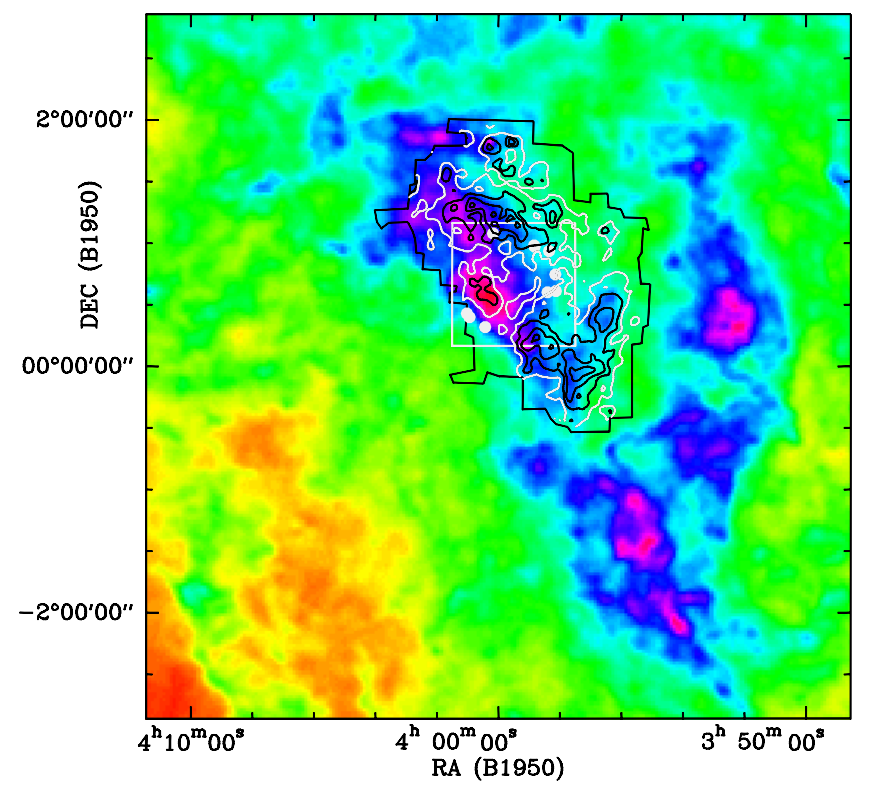

Fig. 5. Map of the IRAS $100 \mu \mathrm{m}$ emission data from NASA/JPLCaltech. Contours: integrated ${ }^{12} \mathrm{CO}(1-0)$ emission $\left(\int T_{\mathrm{A}}^{*} \mathrm{~d} v\right.$; KOSMA observations; lowest contour and step $2 \mathrm{~K} \mathrm{~km} \mathrm{~s}^{-1}$; lowest contour drawn in white). The black irregular outline indicates the mapped region. The white square shows the location and extent of the grism field, and the white filled circles indicate the candidate $\mathrm{H} \alpha$ emission-line stars.

is $1.2 \mathrm{~km} \mathrm{~s}^{-1}$. The total emission in the region observed with the SEST (using $\int T_{\mathrm{mb}} \mathrm{d} v$ ) is $11808.3 \mathrm{~K} \mathrm{~km} \mathrm{~s}^{-1} \operatorname{arcmin}^{2}$, implying $L_{\mathrm{CO}}=14.4 \mathrm{~K} \mathrm{~km} \mathrm{~s}^{-1} \mathrm{pc}^{2}$ for a distance of $120 \mathrm{pc}$. This corresponds to a mass of $56 M_{\odot}\left(X=1.8 \times 10^{20} \mathrm{~cm}^{-2}\left(\mathrm{~K} \mathrm{~km} \mathrm{~s}^{-1}\right)^{-1}\right.$, and including a factor of 1.36 to account for helium).

The ratio of $T_{\mathrm{mb}}{ }^{12} \mathrm{CO}(2-1)$ to $(1-0)$ is about 1.0 (however, too few positions are observed in $(2-1)$ to convolve these data to the (1-0) angular resolution).

Figure 8 shows the distribution of the ${ }^{13} \mathrm{CO}(1-0)$ emission of the northeastern peak, observed with the SEST. This region contains one main peak and several smaller peaks, some of which are located along a ridge. The line width at the positions with the strongest emission is $0.75 \mathrm{~km} \mathrm{~s}^{-1}$. The ${ }^{13} \mathrm{CO}(1-0)$ emission is much less extended than the ${ }^{12} \mathrm{CO}(1-0)$ emission (cf. Fig. 6), a consequence of the low opacity of MBM 18 and clouds like it. Assuming LTE and $T_{\mathrm{ex}}=10 \mathrm{~K}$, we find a mass of $5 M_{\odot}$ from the ${ }^{13} \mathrm{CO}$ observations (or $\sim 10 \%$ of the mass derived from the ${ }^{12} \mathrm{CO}$ data), and ${ }^{13} \mathrm{CO}$ optical depths of typically a few tenths, with a maximum of 0.6 in the core near offset 0,0 (see Fig. 9). The ${ }^{13} \mathrm{CO}$ column density at the location of peak optical depth is about $3.2 \times 10^{15} \mathrm{~cm}^{-2}$. If we apply the standard relation $N\left(\mathrm{H}_{2}\right)=5 \times 10^{5} N\left({ }^{13} \mathrm{CO}\right)$ (Dickman \& Clemens 1983) also to translucent clouds, the column density of $\mathrm{H}_{2} \approx 1.6 \times 10^{21} \mathrm{~cm}^{-2}$. Because $N\left(\mathrm{H}_{2}\right)=10^{21} \times A_{\mathrm{V}}$ (Bohlin et al. 1978, and assuming the gas-to-dust ratio in MBM 18 is the same as for the local interstellar medium), this implies a visual extinction $A_{\mathrm{V}}$ of about $1.6 \mathrm{mag}$ at the peak of the ${ }^{13} \mathrm{CO}$ core. The visual extinction can be derived independently of the Schlegel et al. (1998) colour excess data (cf. Fig. 1) and the standard value of the total-toselective absorption ratio $R_{\mathrm{V}}=E(B-V) / A_{\mathrm{V}}$ of 3.1. At the position of the ${ }^{13} \mathrm{CO}$ core, over a circular region with a diameter of 6 '. 1 , we find $A_{\mathrm{V}} \approx 1.8 \mathrm{mag}$.

\subsection{Effelsberg $-\mathrm{NH}_{3}$}

The $\mathrm{NH}_{3}(1,1)$ line was observed and detected near the core of the cloud area that was mapped in CO with the SEST. The $\mathrm{NH}_{3}$ emission is plotted superimposed on the ${ }^{13} \mathrm{CO}(1-0)$ emission in Fig. 9. The strongest $\mathrm{NH}_{3}$ emission is slightly displaced from the ${ }^{13} \mathrm{CO}$ peak. Line parameters of the average spectrum of the three strongest positions are given in Table 2, together with the parameters of other lines observed at that and other positions. A fit to the hyperfine components indicates that the optical depth of the line is small $(<0.1)$. The line width corrected for hyperfine components is $0.64 \pm 0.08 \mathrm{~km} \mathrm{~s}^{-1}$. The fact that $\mathrm{NH}_{3}$ has been detected suggests that fairly dense material is present, which means that at least some of the necessary conditions for star formation are present in this cloud. (Note that CS was not detected, however). The rotation temperature, derived from the $\mathrm{NH}_{3}(1,1)$ and $(2,2)$ results, following Harju et al. (1993) is $39 \pm 10 \mathrm{~K}$, and the $\mathrm{NH}_{3}$ column density $(1.4 \pm 0.4) \times 10^{13} \mathrm{~cm}^{-2}$.

\subsection{Medicina $-\mathrm{H}_{2} \mathrm{O}$ masers}

All data were reduced with the CLASS-package. Polynomial baselines were removed and spectra were averaged for each position. The results are shown in Table 3. The spectra taken towards Ha7 in Oct. 2008 and May 2009 showed a possible detection when smoothed to a lower resolution (see Fig. 10); results of a Gaussian-fit to the average spectra are reported in the last two lines of Table 3 . To confirm the reality of this feature we reobserved this position in January 2011 for $45 \mathrm{~min}$ on-source. No signal was detected; averaging all spectra pertaining to $\mathrm{Ha} 7$, including those from 2011, shows no detection. Water masers are notoriously variable, especially those associated with lowermass stars (Felli et al. 2007, and references therein) and this nondetection more than 1.5 years after the original observation does not imply that the tentative detection of maser emission in the $2008 / 2009$ observations is not real.

\section{Discussion}

\subsection{Stars}

We derived more accurate spectral types (for the M-stars) from our spectra using the relation $S_{\mathrm{p}}=-10.775(\mathrm{TiO} 5)+8.200$ (Cruz \& Reid 2002; see their Fig. 3 and Table 4), with TiO5 a spectral index describing the strength of the $\mathrm{TiO}$ band at 7128-7135 $\AA$, compared with the continuum at 7042-7046. The uncertainty of the spectral types derived using this relation is 0.5 subtype. The results are presented in Table 4 . The other columns in this table contain the following information: the star name (Col. 1); an indication of whether $\mathrm{H} \alpha$ emission is (Y) or is not $(\mathrm{N})$ present (Col. 2); if "Y", Col. 3 gives the equivalent width of this line. Col. 4 gives an indication $(\mathrm{Y} / \mathrm{N})$ of the presence of the LiI $6708 \AA$ absorption line. In Cols. 5-10 we give the $K$-magnitude, the $(H-K)$ and $(J-H)$ colours, and their uncertainties, respectively. Magnitude and colours are originally from the 2MASS survey (see Table 1), and were transformed to the Bessel \& Brett (1988) system using Eqs. (A1)-(A4) in Carpenter (2001). Finally, Cols. 11-13 list the spectral types, the extinction in the $K$-band $\left(A_{K}\right)$, and the distance of the stars, respectively. Several of these parameters will be determined in the remainder of this section.

As described in Sect. 3.6, the wavelength calibration of the spectra was corrected using measurements of sky lines. After this, the $\mathrm{H} \alpha$ lines in the spectra do not always lie exactly at 

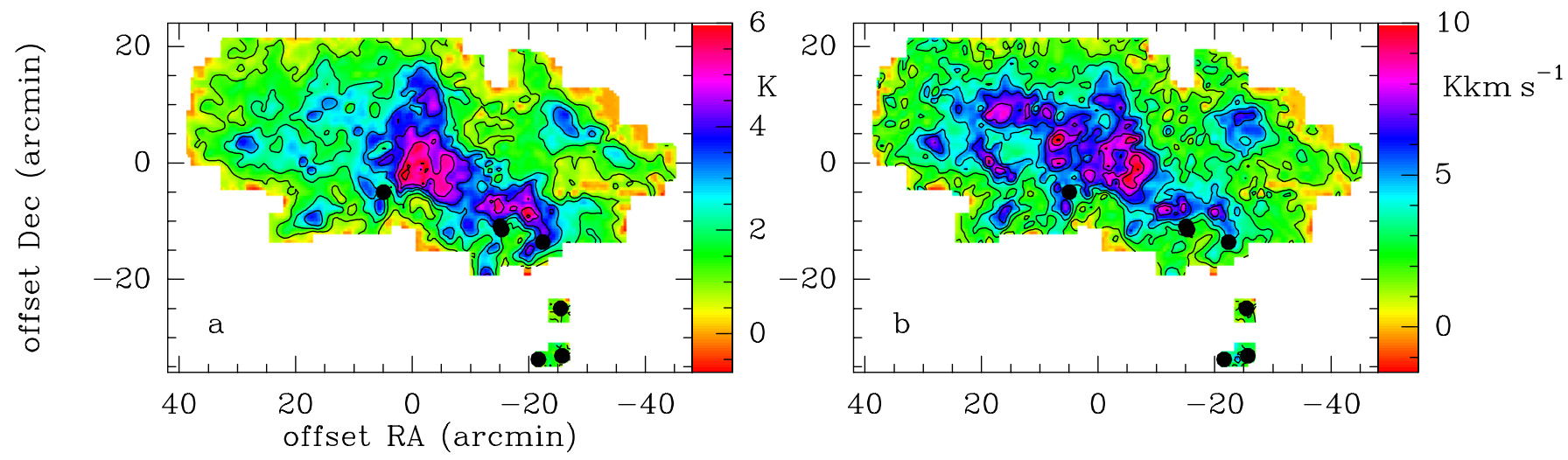

Fig. 6. a) Distribution of peak $T_{\mathrm{A}}^{*}$ of ${ }^{12} \mathrm{CO}(1-0)$ towards LDN 1569 obtained from SEST observations obtained from Gaussian fits to the spectra. Contour levels are $1-6 \mathrm{~K}$ in steps of $1 \mathrm{~K}$. b) As a), but for the line-integrated emission $\int T_{\mathrm{A}}^{*} \mathrm{~d} v$. Contour levels are $1.5-10.5 \mathrm{~K} \mathrm{~km} \mathrm{~s}^{-1}$ in steps of $1.5 \mathrm{~K} \mathrm{~km} \mathrm{~s}^{-1}$. The filled circles indicate the positions of the candidate emission-line stars. Offsets as in Fig. 2.

the rest frequency. Any remaining difference between observed and laboratory wavelengths is due to the radial velocities of the stars with respect to the telescope at the time of observation. For the $\mathrm{H} \alpha$ line this difference ranges from $-1.3 \AA$ (Ha8) to $+1.1 \AA$ (Ha6); Ha1 and $\mathrm{Ha} 7$ have no clearly visible $\mathrm{H} \alpha$ emission or absorption, and an offset could not be determined.

Clear $\mathrm{H} \alpha$ emission was detected in all stars except $\mathrm{Ha} 1$ and Ha7. The wavelength region around LiI is shown in more detail in Fig. 11. Here we have adjusted the wavelength scale by forcing the observed $\mathrm{H} \alpha$ line to its rest frequency (except for $\mathrm{Ha} 1$ and $\mathrm{Ha} 7$, the spectra of which have no $\mathrm{H} \alpha$ line). None of the stars show strong LiI-absorption; there are perhaps hints of the line in $\mathrm{Ha} 5, \mathrm{Ha} 7$ and $\mathrm{Ha}$, although the spectrum of latter star is rather noisy.

Optical spectra of T Tauri stars show a stellar absorption line spectrum, resembling that of a late-type (K or $M)$ dwarf or subgiant photosphere, superimposed by an emission line spectrum (Appenzeller \& Mundt 1989). Furthermore, a distinguishing characteristic of all T Tauri stars is strongly enhanced LiI absorption (weak or absent in most evolved late-type stars; Appenzeller $\&$ Mundt 1989, and references therein). It is clear that the stars observed by us are not T Tauri stars, but M-type dwarf stars.

Both M-dwarfs and T Tauri stars (especially weak-lined ones, i.e. $\mathrm{H} \alpha$ absolute equivalent width less than $\sim 10 \AA$ ) often show X-ray emission (e.g. Neuhäuser et al. 1995). The ROSAT All-Sky Survey data for MBM 18 were analysed by Hearty (1997) following the procedure discussed by Neuhäuser et al. 1995). For two of our stars Hearty found an X-ray counterpart: Ha5 (RXJ0401.4+0106) and Ha8 (RXJ0403.0+0027a). Only the latter appears also in the Second ROSAT PSPC Catalog (ROSAT 2000; 2RXP J040300.2+002748) and in the WGA-catalogue (White et al. 2000; 1WGA J043.0+0027). The X-ray counterpart of Ha5 is apparently just below the detection threshold established by these catalogues. Hearty (1997) obtained an optical spectrum of Ha5 and classified it as a type M3, similar to what we find (Table 4).

The non-T Tauri nature of our target stars is confirmed by comparing their location with those of the young stellar objects (YSOs) in MBM 12, in a colour-colour diagram constructed using WISE observations, shown in Fig. 12. The WISE mission (Wright et al. 2010) has made a sensitive all-sky survey in four mid-IR bands. In a colour-colour plot as shown in Fig. 12 (cf. Fig. 12 in Wright et al. 2010), stars (and early-type galaxies) have colours near zero, spirals are red in the [4.6]-[12.0] colour, while other extra-galactic objects such as Seyfert galaxies and active galactic nuclei are red in both colours. In Fig. 12 we plot the colours of point sources seen projected against all clouds in the MBM-catalogue as grey dots. These point sources fall into two broad categories: stars and galaxies; because of the high (absolute) galactic latitude of the MBM-clouds and their low extinction, it is not a surprise that we find so many extra-galactic objects at those locations. The $\mathrm{H} \alpha$-stars from Table 1 are plotted as diamonds. All lie in the colour range of normal stars, except perhaps for $\mathrm{Ha} 4$, which however has a lower limit to the magnitude in the $12 \mu \mathrm{m}$ band, and thus an upper limit to the [4.6]-[12.0] colour (we will further remark on this star below), as do Ha3 and Ha10 (neither of them was observed spectroscopically by us). As a comparison we also show the locations of the certified YSOs in MBM 12 (Luhman 2001; circles), which have a very different distribution from the stars we observed in MBM 18. This confirms that the MBM 18 stars do not have YSO characteristics, as is indeed borne out by their optical spectra.

In Fig. 13 we plot the locations of the target stars in the infrared colour-colour diagram. The main sequence and the giant branch are taken from Bessel \& Brett (1988). As in Fig. 12, the location of $\mathrm{Ha} 4$ is peculiar. Its spectrum is that of a normal mainsequence star, and as such it should lie between the two dotted lines that indicate the normal reddening band. We can exclude that Ha4 is a galaxy, because the offset of the $\mathrm{H} \alpha$ line from its rest-wavelength $(0.87 \AA)$ indicates a velocity with respect to the Local Standard of Rest of about $-90 \mathrm{~km} \mathrm{~s}^{-1}$. It could be a singleline spectroscopic binary: the companion is too weak to show up in the spectrum of the primary, but does affect the colours.

From this plot we can also obtain an estimate of the spectral types of the stars. These are found to agree with those determined from the relative strength of the $\mathrm{TiO}$ band, except for $\mathrm{Ha} 2$ and $\mathrm{Ha} 8$, for which Fig. 13 suggests M1 and M2, respectively. In the following we use the spectral types as given in Table 4, although the results do not change significantly (not even for $\mathrm{Ha} 2$ and $\mathrm{Ha} 8$ ) if we use those derived from Fig. 13 instead.

From the observed and intrinsic colours (Cox 2000) we find the colour excess and thus the extinction, by averaging $A_{\mathrm{V}}=15.87 \mathrm{E}(H-K)$ and $A_{\mathrm{V}}=9.35 \mathrm{E}(J-H)$; furthermore $A_{K}=0.112 A_{\mathrm{V}}$ (Rieke \& Lebofsky 1985). As can be seen from Fig. 13, the M-type stars are all very close to the main-sequence locus and the extinction will therefore be very small, and in fact for several stars we found the two colour excesses very close to zero but of opposite sign. In these cases we assumed $A_{\mathrm{V}}=A_{K}=$ 0 . The distance modulus for a star with observed magnitude $m_{K}$ and absolute magnitude $M_{K}$ is $m_{K}-M_{K}=5 \log d-5+A_{K}$, from 


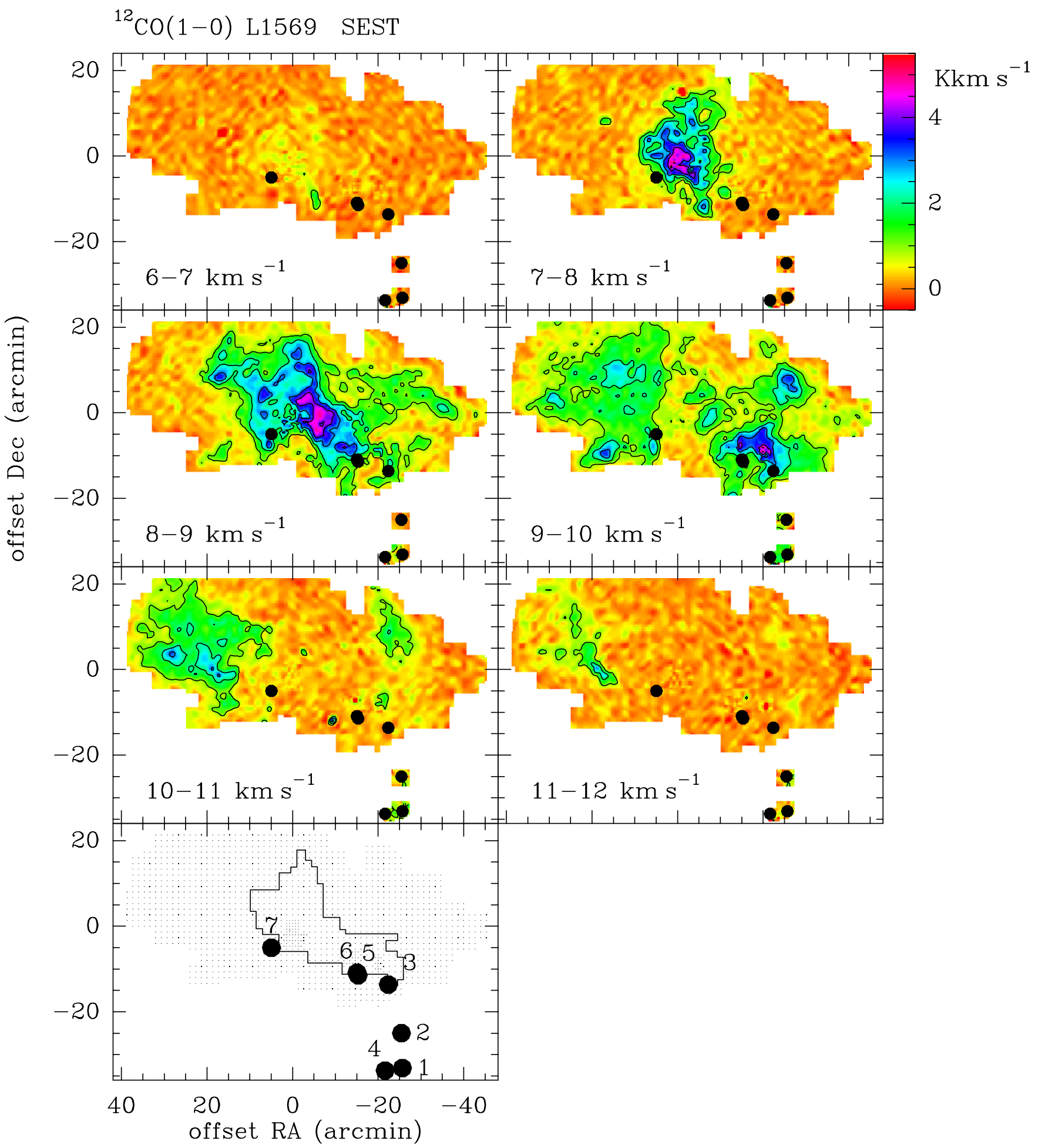

Fig. 7. SEST observations. ${ }^{12} \mathrm{CO}(1-0)$ line emission $\int T_{\mathrm{A}}^{*} \mathrm{~d} v$ integrated over velocity intervals of $1 \mathrm{~km} \mathrm{~s}^{-1}$ between 6 and $12 \mathrm{~km} \mathrm{~s}^{-1}$. Lowest contour level and step are $1 \mathrm{~K} \mathrm{~km} \mathrm{~s}^{-1}$. The observed positions are indicated in the lower panel. This panel also indicates the area observed with the SEST in ${ }^{13} \mathrm{CO}(1-0)$. The filled circles indicate the positions of the candidate emission-line stars, which are labelled with their numbers as in Table 1. Offsets as in Fig. 2.

which we can derive the distance $d$. The absolute $M_{K}$ magnitudes for the various M-type stars were found from the intrinsic $(V-K)$-colour (Cox 2000) and the absolute visual magnitude $M_{\mathrm{V}}$ (Schmidt-Kaler 1982).

The results are summarised in Table 4. The first thing we notice is the wide range in distances of the stars, from 60 to $250 \mathrm{pc}$, and the low extinction values. The distance to
MBM 18 has been determined by several authors, using a variety of methods such as star counts (Magnani \& de Vries 1986; Chaplin et al. 2004), photometry (Franco 1988; Penprase 1992), and spectroscopy of interstellar absorption lines (Penprase et al. 1990; Penprase 1993). It should be noted that virtually none of the stars studied in the literature are seen projected against that part of the cloud that has been detected in $\mathrm{CO}$, which is 

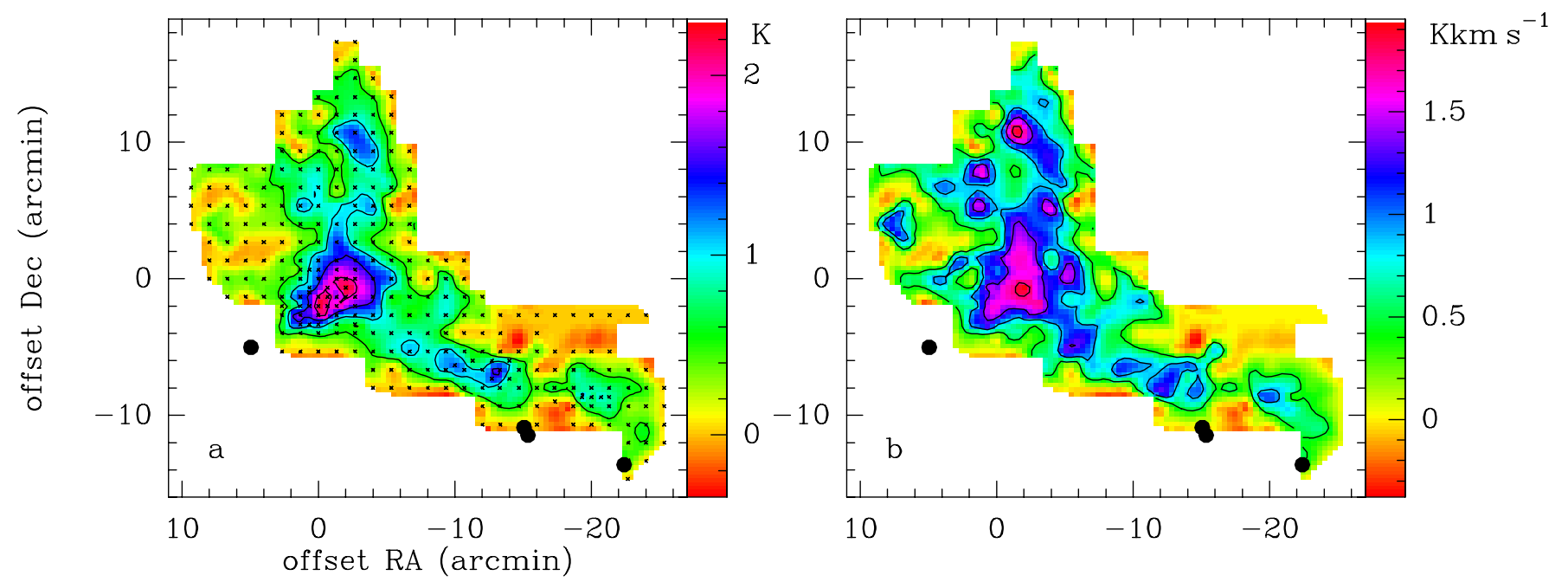

Fig. 8. a) Distribution of peak $T_{\mathrm{A}}^{*}$ of ${ }^{13} \mathrm{CO}(1-0)$ towards LDN 1569 obtained from SEST observations and Gaussian fits to the spectra. Contour levels are 0.5 to $2.0 \mathrm{~K}$ in steps of $0.5 \mathrm{~K}$. The crosses indicate the observed positions. b) As a), but for the line integrated emission $\int T_{\mathrm{A}}^{*} \mathrm{~d} v$. Contour levels are 0.45 to $1.9 \mathrm{~K} \mathrm{~km} \mathrm{~s}^{-1}$ in steps of $0.45 \mathrm{~K} \mathrm{~km} \mathrm{~s}^{-1}$. The filled circles indicate the positions of the candidate emission-line stars. Offsets as in Fig. 2.

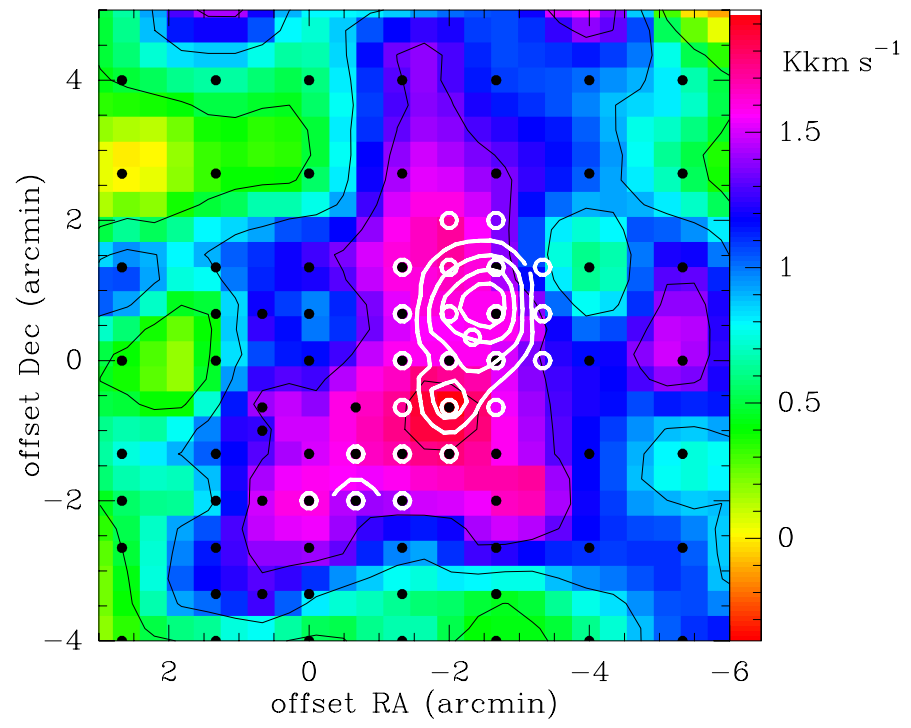

Fig. 9. a) Distribution of integrated $\mathrm{NH}_{3}(1,1)$ emission (white contours) towards LDN 1569 (Effelsberg data) superposed on the integrated ${ }^{13} \mathrm{CO}(1-0)$ emission (colour plot; black contours) observed with the SEST. The $\mathrm{NH}_{3}$ contour levels are $0.05(0.05) 0.20 \mathrm{~K} \mathrm{~km} \mathrm{~s}^{-1}\left(\int T_{\mathrm{mb}} \mathrm{dv}\right)$ and the ${ }^{13} \mathrm{CO} \int T_{\mathrm{A}}^{*} \mathrm{~d} v$ contour levels $0.45(0.45) 1.8 \mathrm{~K} \mathrm{~km} \mathrm{~s}^{-1}$. Black dots indicate positions observed in ${ }^{13} \mathrm{CO}$ and white circles those observed in $\mathrm{NH}_{3}$.

where our target stars are located (in projection). Most authors agree more or less on a distance of $120-150 \mathrm{pc}$, but the cloud has "a patchy and highly irregular structure" (Penprase 1993). From a plot of visual extinction as a function of distance, Penprase (1992) concluded that the MBM 18/LDN 1569 complex extends along the line-of-sight from about $140 \mathrm{pc}$ to $300 \mathrm{pc}$. Regarding this latter statement, we note that it is highly unlikely that, as a molecular entity, MBM 18/LDN 1569 is a few parsec wide and $160 \mathrm{pc}$ long, with the long axis aligned almost exactly along the line-of-sight. The region containing MBM 18 and MBM 16 (see Fig. 1) lies south of the Taurus-Aurigae dark cloud complexes, which have several absorbing layers at different distances (e.g., Magnani 1988). It is likely that this complex distribution
Table 2. Line parameters

\begin{tabular}{lcccccc}
\hline \hline Line & \multicolumn{2}{c}{ Offset } & $T_{\mathrm{mb}}$ & $V_{\mathrm{lsr}}$ & $\Delta V$ & $\int T_{\mathrm{mb}} \mathrm{d} v$ \\
& $\mathrm{RA}$ & $\begin{array}{c}\mathrm{Dec} \\
\text { arcmin }\end{array}$ & $\mathrm{K}$ & $\mathrm{km} \mathrm{s}^{-1}$ & $\mathrm{~km} \mathrm{~s}^{-1}$ & $\mathrm{~K} \mathrm{~km} \mathrm{~s}^{-1}$ \\
\hline $\mathrm{CO}(1-0)^{a}$ & -2.7 & 0.7 & 7.35 & 7.93 & 1.38 & 10.79 \\
${ }^{13} \mathrm{CO}(1-0)^{a}$ & -2.7 & 0.7 & 2.40 & 7.79 & 0.91 & 2.31 \\
$\mathrm{CO}(2-1)^{a}$ & -1.3 & -1.3 & 4.94 & 8.00 & 2.18 & 11.50 \\
${ }^{13} \mathrm{CO}(2-1)^{a}$ & -1.3 & -1.3 & 1.44 & 7.90 & 1.15 & 1.77 \\
$\mathrm{C}^{18} \mathrm{O}(1-0)^{a}$ & -1.3 & -1.3 & 0.31 & 7.72 & 0.39 & 0.13 \\
$\mathrm{CS}(2-1)^{a}$ & -1.3 & -1.3 & $\ldots$. & $\ldots$ & $\ldots$ & $\ldots$ \\
${ }^{13} \mathrm{CO}(2-1)^{a}$ & 0.0 & 0.0 & 1.31 & 7.96 & 1.13 & 1.57 \\
${ }^{13} \mathrm{CO}(2-1)^{a}$ & -13.3 & -6.7 & 0.67 & 9.24 & 0.89 & 0.63 \\
$\mathrm{CO}(2-1)^{b}$ & 0.0 & 0.0 & 4.74 & 7.88 & 1.16 & 5.87 \\
$\mathrm{CO}(2-1)^{b}$ & 9.37 & -32.93 & 0.72 & 11.20 & 1.53 & 1.17 \\
$\mathrm{CO}(2-1)^{b}$ & 5.98 & -36.30 & 1.44 & 10.68 & 1.08 & 1.66 \\
$\mathrm{CO}(2-1)^{b}$ & 2.58 & -39.67 & 1.10 & 10.88 & 1.71 & 1.99 \\
$\mathrm{NH}_{3}(1,1)^{c}$ & -2.3 & 0.5 & 0.20 & 7.88 & 0.91 & 0.19 \\
$\mathrm{NH}_{3}(2,2)^{c}$ & -2.3 & 0.5 & 0.05 & 7.82 & 0.27 & 0.02 \\
\hline
\end{tabular}

Notes. ${ }^{(a)}$ SEST data. ${ }^{(b)}$ JCMT data. ${ }^{(c)}$ Effelsberg data, average spectra of the strongest 3 positions. ${ }^{(\dagger)} \mathrm{rms}=0.064 \mathrm{~K}$.

of absorbing layers continues south of Taurus into the region containing MBM 18 and MBM 16. Those clouds are likely associated with just one of those absorbing layers.

On the other hand, from a similar study as that by Penprase (1992) a considerably lower value $(80 \pm 20 \mathrm{pc})$ was found by Chaplin et al. (2004). Magnani \& de Vries (1986) from a comparison of theoretical and observed Wolf diagrams found a best fit for a distance of $50 \mathrm{pc}$ (and an upper limit of $175 \mathrm{pc}$ ). In view of the above, all stars in Table 4 are apparently located at least in the extended complex that Penprase (1993) refers to; but $\mathrm{Ha} 1, \mathrm{Ha} 8$, and perhaps also $\mathrm{Ha} 2$, lie at the distance that the bulk of the molecular material of MBM 18 is located at. The visual extinction of our targets stars has a maximum value of about $0.5 \mathrm{mag}$, which is a typical average value for the cloud (e.g., Penprase 1993). Four of the stars have no measurable extinction, among which $\mathrm{Ha} 7$ at $252 \mathrm{pc}$ is the most distant one in our sample. 


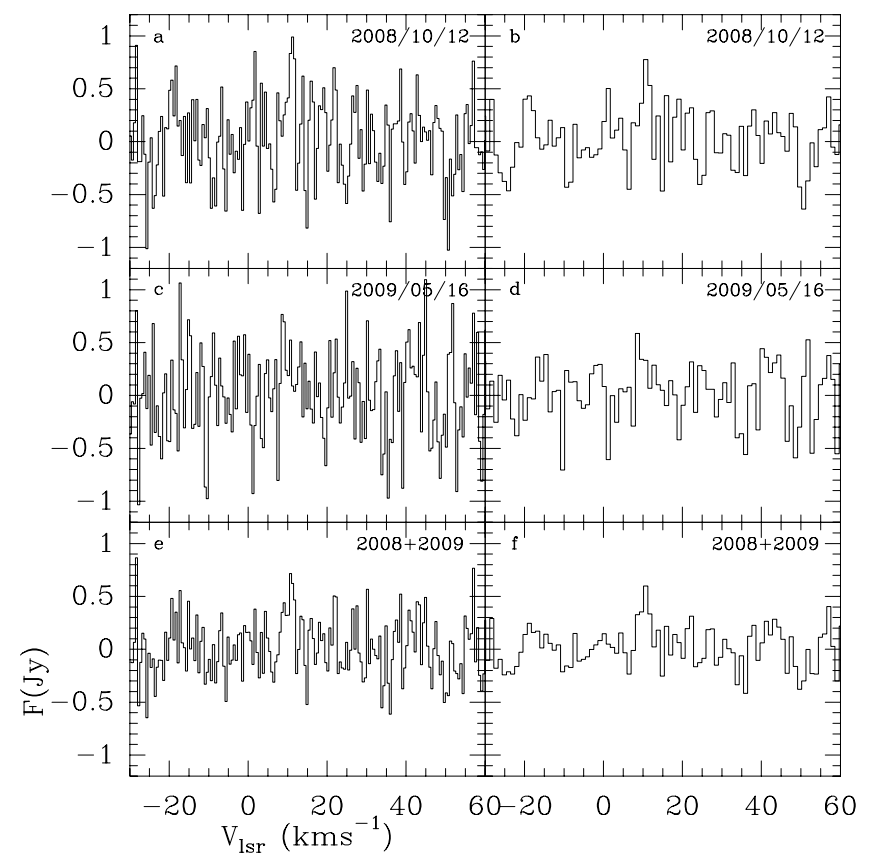

Fig. 10. a) October 2008 spectrum at $22 \mathrm{GHz}$ of candidate $\mathrm{H} \alpha$ emission-line star Ha7. The spectrum is smoothed to a resolution of $0.53 \mathrm{~km} \mathrm{~s}^{-1}$. b) As a), but smoothed to a resolution of $1.05 \mathrm{~km} \mathrm{~s}^{-1}$. c) As a), but for the May 2009 spectrum. d) As b), but for the May 2009 spectrum. e) Average spectrum at $22 \mathrm{GHz}$ of the 2008 and 2009 observations of $\mathrm{Ha} 7$, smoothed to a resolution of $0.53 \mathrm{~km} \mathrm{~s}^{-1}$. f) As e), but smoothed to a resolution of $1.05 \mathrm{~km} \mathrm{~s}^{-1}$.

When comparing the photometry of our stars with the premain-sequence isochrones of Palla \& Stahler (1999) we obtain a good fit for values of distances, extinction, and masses in accordance with the values listed in Table 4. These fits show that the stars have ages between 7.5 and $15 \mathrm{Myr}$ : young enough to fit on pre-main-sequence isochrones, but old enough for Li to have disappeared (in low-mass stars lithium is effectively destroyed by convective mixing on timescales of a few Myr). Considering the unbound state of the molecular gas (see Sect. 4.2), these stars are too old to have been formed in MBM 18, as presently constituted.

We thus find at least four active late-type stars in our grism field, with spectral types down to dM4. We can derive the space density of M-dwarfs of type dM0-dM4 from the luminosity function of M-dwarfs, derived by Zheng et al. (2001) from HST star counts at $|b|>17^{\circ}$. According to Joy \& Abt (1974), $11 \%$ of stars in that range of spectral types are dMe. In the volume of space sampled by us in the 1 sq. degree grism field (a truncated cone between 80 and $300 \mathrm{pc}$ - which is roughly the extent of molecular gas along the line-of-sight towards MBM 18), we therefore expect about $6 \mathrm{dMe}$ stars of type $\mathrm{dM} 0-\mathrm{dM} 4$, which agrees with our observations.

\subsection{Molecular gas}

Using the KOSMA ${ }^{12} \mathrm{CO}$ data, we determined the virial state of the cloud. The number of detections (499) multiplied by the area of a map-element $\left(4 \times 4 \operatorname{arcmin}^{2}\right)$ can be used as an estimate of the cloud area. The "effective radius" of the cloud is then $r_{\mathrm{e}}=\sqrt{(\mathrm{area} / \pi)}$; for a representative distance of $120 \mathrm{pc}$ we find $r_{\mathrm{e}}=1.8 \mathrm{pc}$. Gaussian profiles were fitted to the 499 detections and the variance of the velocity centroids was $0.88 \mathrm{~km} \mathrm{~s}^{-1}$, equivalent to a $\Delta V(F W H M)$ of $2.1 \mathrm{~km} \mathrm{~s}^{-1}$. For a density distribution $\rho \propto r^{-2}, M_{\text {vir }}\left(M_{\odot}\right)=126 r_{\mathrm{e}} \Delta V^{2}$, where $r_{\mathrm{e}}$ is the radius
Table 3. Medicina $\mathrm{H}_{2} \mathrm{O}$ maser observations.

\begin{tabular}{|c|c|c|c|c|c|}
\hline Position & $\begin{array}{c}\text { Date } \\
(y-m-d)\end{array}$ & $\begin{array}{r}\mathrm{rms}^{1} \\
(\mathrm{Jy})\end{array}$ & $\begin{array}{c}F \\
(\mathrm{Jy})\end{array}$ & $\begin{array}{c}\Delta V \\
\left(\mathrm{~km} \mathrm{~s}^{-1}\right)\end{array}$ & $\begin{array}{c}V_{\mathrm{lsr}} \\
\left(\mathrm{km} \mathrm{s}^{-1}\right)\end{array}$ \\
\hline \multirow[t]{3}{*}{ Ha1 } & 2008-10-11 & 1.14 & $\ldots$ & $\ldots$ & $\ldots$ \\
\hline & 2009-05-16 & 1.74 & $\ldots$ & $\ldots$ & $\ldots$ \\
\hline & 2011-02-20 & 1.55 & $\ldots$ & $\ldots$ & $\ldots$ \\
\hline \multirow[t]{4}{*}{$\mathrm{Ha} 2$} & 2008-10-12 & 1.02 & $\ldots$ & $\ldots$ & $\ldots$ \\
\hline & 2009-05-16 & 1.08 & $\ldots$ & $\ldots$ & $\ldots$ \\
\hline & 2011-02-20 & 1.69 & $\ldots$ & $\ldots$ & $\ldots$ \\
\hline & 2011-03-18 & 1.13 & $\ldots$ & $\ldots$ & $\ldots$ \\
\hline \multirow[t]{5}{*}{$\mathrm{Ha} 5$} & 2008-10-10 & 1.12 & $\ldots$ & $\ldots$ & $\ldots$ \\
\hline & 2009-04-02 & 1.35 & $\ldots$ & $\ldots$ & $\ldots$ \\
\hline & 2009-05-16 & 0.99 & $\ldots$ & $\ldots$ & $\ldots$ \\
\hline & 2011-02-20 & 1.28 & $\ldots$ & $\ldots$ & $\ldots$ \\
\hline & 2011-03-18 & 0.60 & $\ldots$ & $\ldots$ & $\ldots$ \\
\hline \multirow[t]{2}{*}{ Ha6 } & 2009-05-16 & 1.07 & $\ldots$ & $\ldots$ & $\ldots$ \\
\hline & 2011-02-20 & 1.42 & $\ldots$ & $\ldots$ & $\ldots$ \\
\hline \multirow[t]{5}{*}{ Ha7 } & 2008-10-12 & 0.92 & $\ldots$ & $\ldots$ & $\ldots$ \\
\hline & 2009-05-16 & 1.10 & $\ldots$ & $\ldots$ & $\ldots$ \\
\hline & 2011-01-19 & 0.36 & $\ldots$ & $\ldots$ & $\ldots$ \\
\hline & 2011-02-20 & 1.24 & $\ldots$ & $\ldots$ & $\ldots$ \\
\hline & 2011-03-18 & 1.02 & $\ldots$ & $\ldots$ & $\ldots$ \\
\hline \multirow[t]{3}{*}{$\mathrm{Ha} 8$} & $2008-10-10$ & 0.99 & $\ldots$ & $\ldots$ & $\ldots$ \\
\hline & 2009-05-16 & 1.08 & $\ldots$ & $\ldots$ & $\ldots$ \\
\hline & 2011-02-20 & 2.13 & $\ldots$ & $\ldots$ & $\ldots$ \\
\hline \multirow[t]{2}{*}{$\mathrm{Ha} 9$} & 2008-10-11 & 1.51 & $\ldots$ & $\ldots$ & $\ldots$ \\
\hline & 2009-05-16 & 1.03 & $\ldots$ & $\ldots$ & $\ldots$ \\
\hline \multirow[t]{2}{*}{ Ha10 } & 2008-10-11 & 1.27 & $\ldots$ & $\ldots$ & $\ldots$ \\
\hline & 2009-05-16 & 1.08 & $\ldots$ & $\ldots$ & $\ldots$ \\
\hline $\mathrm{Ha}^{2}$ & $2008+09$ & 0.28 & 0.55 & $2.78 \pm 0.90$ & $10.46 \pm 0.41$ \\
\hline $\mathrm{Ha}^{3}$ & $2008+09$ & 0.18 & 0.53 & $2.88 \pm 0.89$ & $10.45 \pm 0.42$ \\
\hline
\end{tabular}

Notes. ${ }^{(1)}$ rms noise per channel of width $0.132 \mathrm{~km} \mathrm{~s}^{-1}$. (2) Line parameters from a Gaussian fit to the average of the spectra from 2008 and 2009 , smoothed to a resolution of $0.53 \mathrm{~km} \mathrm{~s}^{-1}$. (3) As 2, but smoothed to a resolution of $1.05 \mathrm{~km} \mathrm{~s}^{-1}$.

of the cloud in pc and $\Delta V\left(\mathrm{~km} \mathrm{~s}^{-1}\right)$ is a measure of the velocity dispersion along the given line-of-sight. We thus obtain a virial mass for the cloud $M_{\mathrm{vir}} \approx 1000 M_{\odot}$.

In Sect. 3.3 we obtained a mass (from the integrated emission) for the cloud of $162 M_{\odot}$ for a distance of $120 \mathrm{pc}$. It is therefore clear that MBM 18, like most high-latitude clouds, is not gravitationally bound and should be breaking up on the sound crossing time scale $\left(\sim 10^{6}\right.$ years). However, this does not mean that the cloud is incapable of star formation. Gravitationally unbound clouds can contain clumps that are dense enough to be bound, and in which star formation might occur. The wellstudied high-latitude cloud, MBM 12, is not gravitationally bound either (Pound et al. 1990), yet it is a prolific star-forming region with at least a dozen young stellar objects associated with the cloud (Luhman 2001).

We ran CLUMPFIND 5 (Williams et al. 1994) on the KOSMA observations, using $0.6 \mathrm{~K}$ for the lowest contouring value and the increment $(\sim 2.9$ times the median rms in the data see Sect. 2.3.1), and found 12 clumps, with parameters as listed in Table 5. The combined mass in all clumps, as determined from their combined CO-luminosity, is $\sim 116 M_{\odot}$, which is $\sim 72 \%$ of the total mass, determined from all emission (see Sect. 3.3).

In Table 5 we give the following information for each clump: a running number (Col. 1); offsets in Right Ascension and Declination (Cols. 2 and 3) relative to the central position; peak

5 http://www.ifa.hawaii.edu/users/jpw/clumpfind.shtml 
Table 4. Results from the TNG observations for the late-type stars.

\begin{tabular}{|c|c|c|c|c|c|c|c|c|c|c|c|c|}
\hline Star & $\mathrm{H} \alpha$ & $\begin{array}{c}E W(\mathrm{H} \alpha)^{\dagger} \\
\AA\end{array}$ & LiI & $K^{a}$ & $\sigma_{K}$ & $\begin{array}{r}(H-K)^{a} \\
\mathrm{ma}\end{array}$ & $\sigma_{\mathrm{H}-\mathrm{K}}$ & $\begin{array}{r}(J-H)^{a} \\
\operatorname{maq}\end{array}$ & $\sigma_{J-H}$ & Sp.T. & $\begin{array}{c}A_{K} \\
\mathrm{mag}\end{array}$ & $\begin{array}{c}d \\
\mathrm{pc}\end{array}$ \\
\hline $\mathrm{Ha} 1$ & $\mathrm{~N}$ & & $\mathrm{~N}$ & 11.304 & 0.026 & 0.214 & 0.036 & 0.654 & 0.036 & $\mathrm{dM} 2.5$ & 0.0 & 128 \\
\hline $\mathrm{Ha} 2$ & $\mathrm{Y}$ & -1.5 & $\mathrm{~N}$ & 11.076 & 0.023 & 0.210 & 0.032 & 0.710 & 0.034 & $\mathrm{dM} 3.5 \mathrm{e}$ & 0.0 & 109 \\
\hline $\mathrm{Ha} 5$ & $\mathrm{Y}$ & -4.0 & Y? & 10.044 & 0.021 & 0.290 & 0.032 & 0.634 & 0.034 & $\mathrm{dM} 4.0 \mathrm{e}$ & 0.02 & 63 \\
\hline Ha6 & Y & -5.1 & Y? & 10.988 & 0.023 & 0.323 & 0.036 & 0.658 & 0.037 & $\mathrm{dM} 4.0 \mathrm{e}$ & 0.06 & 95 \\
\hline $\mathrm{Ha} 7$ & $\mathrm{~N}$ & & Y? & 12.580 & 0.030 & 0.215 & 0.039 & 0.640 & 0.037 & $\mathrm{dM} 1.5$ & 0.0 & 252 \\
\hline $\mathrm{Ha} 8$ & Y & -3.0 & $\mathrm{~N}$ & 11.390 & 0.026 & 0.191 & 0.036 & 0.633 & 0.035 & $\mathrm{dM} 3.5 \mathrm{e}$ & 0.0 & 128 \\
\hline
\end{tabular}

Notes. ${ }^{(\dagger)}$ By convention negative equivalent widths indicate emission lines. ${ }^{(a)}$ Magnitudes and colours transformed from 2 MASS to the Bessel \& Brett (1988) system using Carpenter (2001).
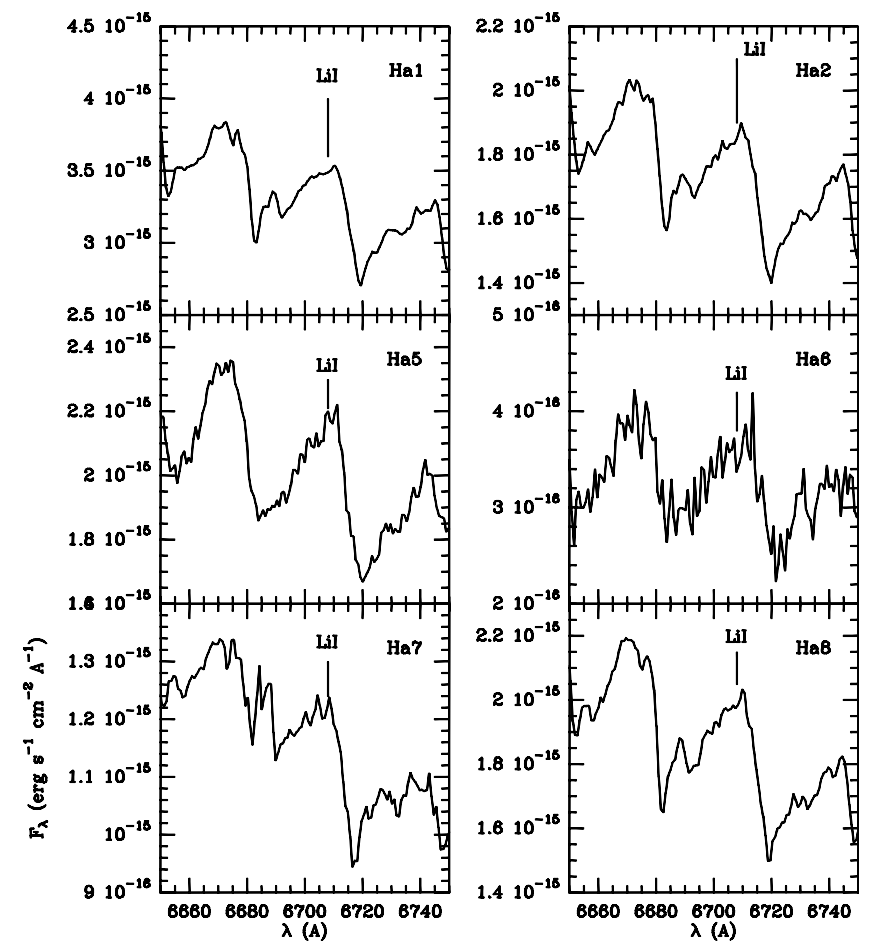

Fig. 11. Detail of the spectra of the targets identified in each panel, showing the wavelength region around LiI (as indicated). For all stars except Ha1 and Ha7 (which have no $\mathrm{H} \alpha$ ), the wavelength scale was adjusted by shifting the $\mathrm{H} \alpha$ line to its rest frequency (see text).

temperature, velocity of the peak, and linewidth (Cols. 4-6); the HWHM-radius $\left(r_{1}\right)$ in arcmin, derived from the dispersion in both coordinates, and corrected for beam size (Col. 7); the clump radius $\left(r_{2}\right)$ in arcmin, derived from the total number of clump pixels above the adopted threshold value, assuming a circular distribution and corrected for beam size (Col. 8). In Cols. 9 and 10 we give $r_{1}$ and $r_{2}$, respectively, in parsec, assuming a distance of $120 \mathrm{pc}$. The CO luminosity, $L_{\mathrm{CO}}$ is in Col. 11 , while the virial masses, assuming a density distribution $\rho \propto r^{-2}$ (i.e., $\left.M_{\mathrm{vir}}\left(M_{\odot}\right)=126 r \Delta V^{2}\right)$, are given in Col. $12\left(r=r_{1}\right)$ and Col. 13 $\left(r=r_{2}\right)$.

Figure 14a shows the virial parameter $\alpha=M_{\mathrm{vir}}\left(r_{1}\right) / M_{\mathrm{CO}}$ as a function of $M_{\mathrm{CO}}=3.9 L_{\mathrm{CO}}$. The virial parameter is a measure of the extent to which a cloud or clump is in equilibrium. Under idealised conditions, $\alpha=1$ for a clump in virial equilibrium, without external pressure and in the absence of a magnetic field; if the kinetic energy of a clump is balanced by its gravitational energy, $\alpha=2$, and the clump is said to be gravitationally bound. In MBM 18 all clumps have $\alpha \gtrsim 6$, thus gravity is not the dominating force in any of these structures.

However, with observations performed with a 3.9 beam on a $4^{\prime}$ raster, the KOSMA map is incompletely sampled, and this may be expected to influence the clump identification process. With the SEST we mapped only the northern part of the cloud, but at higher resolution (although still incompletely sampled). Running CLUMPFIND on these data (using $0.72 \mathrm{~K}[=3 \sigma]$ for the lowest contouring value and increment) results in 24 clumps. The clumps' parameters are given in Table 6 , where the description of the columns is the same as for Table 5. The total mass contained in the clumps (from their combined CO-luminosities) is $15.4 M_{\odot}$, which is only $27 \%$ of the mass determined from all emission in the cloud (Sect. 3.4). Apparently there is much diffuse emission that CLUMPFIND cannot assign to any clumps. For the SEST-clumps we plot the virial parameter $\alpha$ versus the clump mass $M_{\mathrm{CO}}=3.9 L_{\mathrm{CO}}$ in Fig. 14b. Also in these higherresolution data we find that all clumps have $\alpha \gtrsim 5.5$, implying that none of the clumps are gravitationally bound.

\subsection{CO pedestals}

Many of the SEST CO(1-0) profiles show evidence of redand blue-shifted low-level emission "wings" (see Fig. 15). This phenomenon is routinely associated with bipolar outflows and is a hallmark of winds from an embedded YSO (e.g., Bachiller 1996). However, this is not likely the case for MBM 18. No discernible blue- or red-shifted lobes can be traced out in the channel maps, nor can the winged profiles be associated distinctly with any of the candidate T Tauri stars. It is more likely that the observed wings are a manifestation of the $\mathrm{CO}$ "pedestals" that are not associated with star formation in translucent high-latitude clouds (Blitz et al. 1988; Magnani et al. 1990). These pedestals are characterstic of the fragmented structure of these clouds, a likely consequence of turbulence. Shore et al. (2003) and Barriault et al. (2010) proposed that high-latitude clouds may form at the confluence of atomic gas flows. In these scenarios, a turbulent cascade smears out large-scale velocity gradients, leaving only small-scale centroid fluctuations (Shore et al. 2006). Observationally, these small-scale velocity fluctuations manifest themselves as low-level emission at velocities other than the cloud average velocity where the bulk of the emission lies. Figure 16 is the average spectrum of the central $400^{\prime \prime} \times$ $400^{\prime \prime}$ region observed in $\mathrm{CO}(1-0)$ with SEST (Fig. 6). The bulk of the $\mathrm{CO}$ emission is concentrated in a Gaussian profile with $V_{\text {lsr }} \sim 8 \mathrm{~km} \mathrm{~s}^{-1}$. The wings or pedestals are clearly noticeable, 


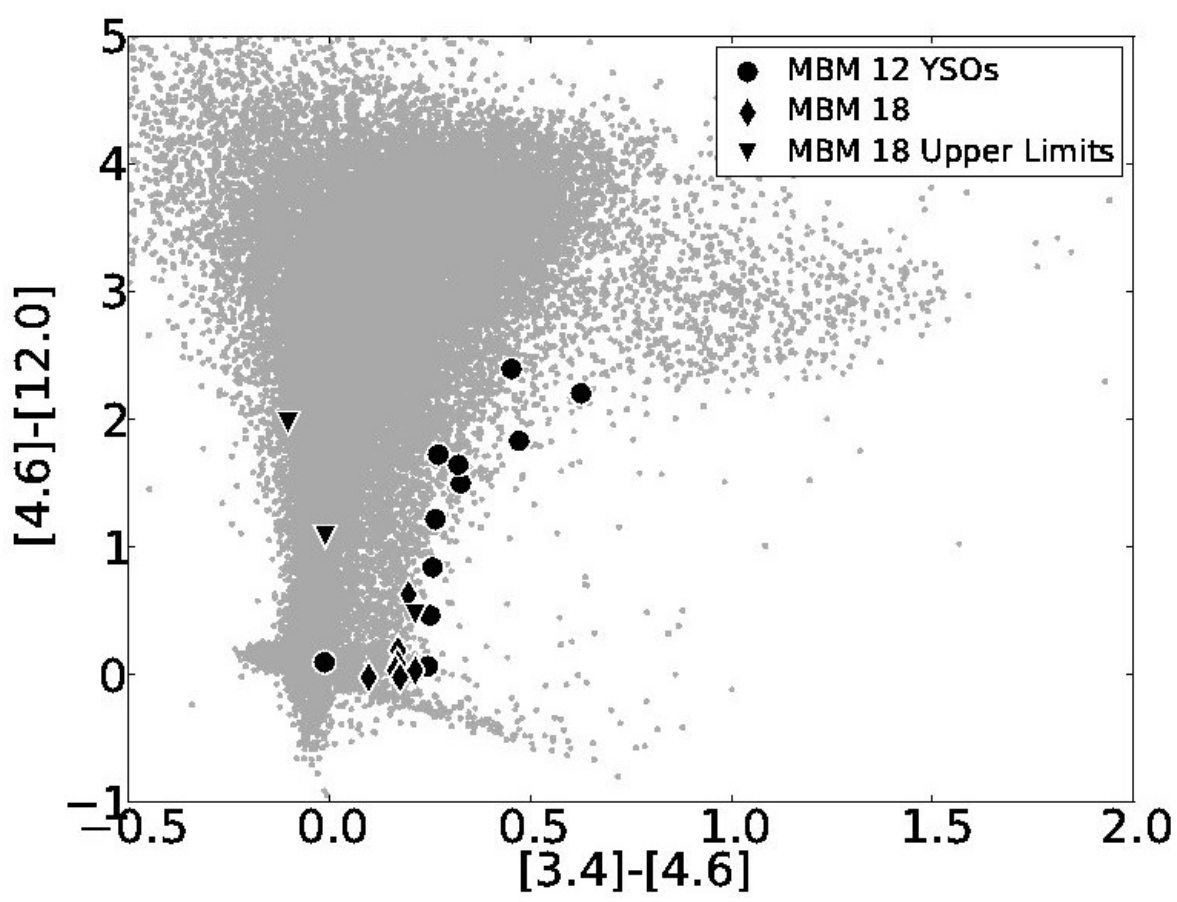

Fig. 12. Colour-colour diagram based on WISE data. The grey dots are point sources seen projected against all clouds in the MBM catalogue. Most in the swarm have the colours of (spiral) galaxies, while stars and elliptical galaxies have colours near zero. The diamonds identify the candidate $\mathrm{H} \alpha$ emission-line stars found in our grism field (Table 1), with upper limits indicated by upside-down triangles. For comparison, the circles indicate the YSOs found in MBM12 (Luhman 2001).

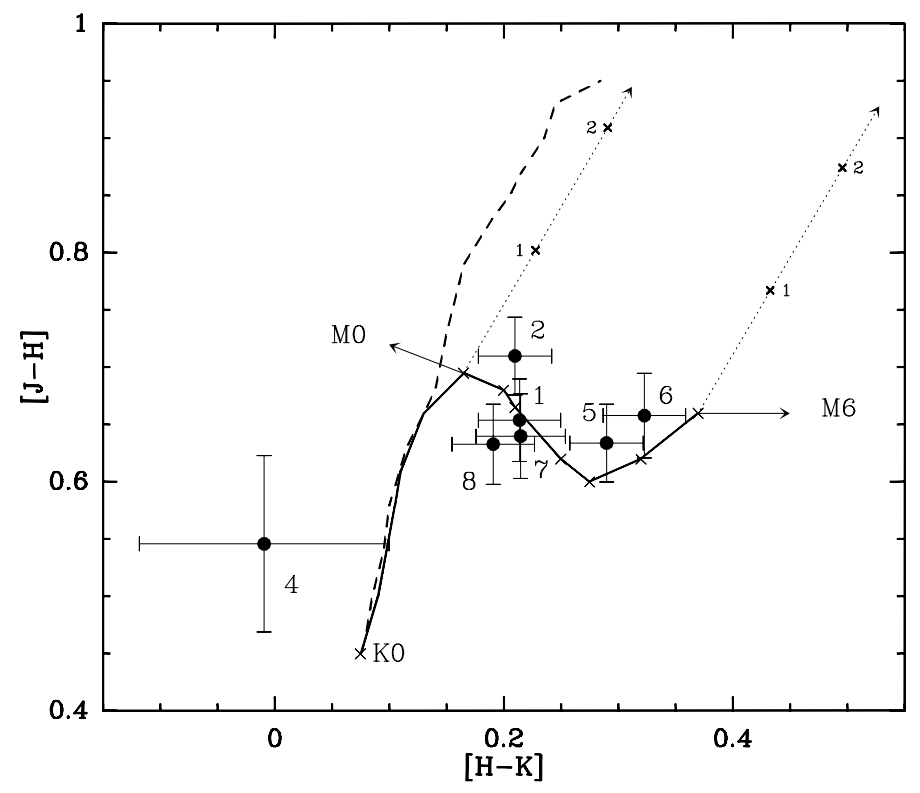

Fig. 13. Colour-colour diagram of the observed stars (2MASS colours transformed to the Bessel \& Brett (1988 system; see text). The intrinsic main sequence (drawn; shown for spectral types $\mathrm{K} 0$ and later) and giant branch (dashed; from type G4 at the bottom to M5 at the top) are taken from Bessel \& Brett (1988). Along the main sequence we indicate the locations of unreddened stars of type M0 V to M6 V with crosses and label two of these. The direction of interstellar reddening is indicated by the dotted lines starting at types M0 and M6, where black crosses indicate values of visual extinction $A_{\mathrm{V}}=1$ and 2 mag.

extending from 4 to $11 \mathrm{~km} \mathrm{~s}^{-1}$. We note that this phenomenon is not intermittency as described by Falgarone \& Phillips (1990), which one would expect to be much weaker and not visible in individual spectra like those shown in Fig. 15. To detect the signature of intermittency, a probability density function (PDF) analysis should be performed, which would require extending our high-resolution observations over the entire cloud. In summary, the lack of any clear bipolar structure to the wing emission, and the absence of correlation of the wings with the positions of the candidate $\mathrm{T}$ Tauri stars indicate that the wings seen in individual and composite profiles of the central region in MBM 18 are not evidence of star formation but, rather, of the fragmented structure of the molecular cloud as a consequence of turbulence.

\section{Conclusions}

The main objective of the observations presented and analysed in this paper, is to investigate whether low-mass star formation occurs in the translucent cloud MBM 18 (LDN 1569).

We have mapped the ${ }^{12} \mathrm{CO}(1-0)$ emission in the high-latitude cloud MBM 18 (LDN 1569) with the KOSMA 3-m telescope; this is the first time this object has been fully mapped in CO. The northern part of the cloud was also mapped at higher resolution in ${ }^{12} \mathrm{CO}(1-0)$ and ${ }^{13} \mathrm{CO}(1-0)$ with the SEST. The total cloud mass, estimated from the integrated $\mathrm{CO}$ emission, is $162 M_{\odot}$, assuming a distance of $120 \mathrm{pc}$ and $X=N\left(\mathrm{H}_{2}\right) / \int T_{\mathrm{mb}} \mathrm{d} v=1.8 \times$ $10^{20} \mathrm{~cm}^{-2}\left(\mathrm{~K} \mathrm{~km} \mathrm{~s}^{-1}\right)^{-1}$. The mapping data show that the cloud contains substantial spatial substructure, while the channel maps also reveal significant velocity structure. The virial mass, as determined from the standard deviation of the central velocities of Gaussian fits to the emission profiles at all detected positions, is at least $10^{3} M_{\odot}$, and thus much larger than the gravitational mass. This implies that MBM 18 is not gravitationally bound. We used CLUMPFIND to analyse these substructures and found 12 clumps (KOSMA data), with beam-corrected equivalent (HWHM) radii ranging from $0.19 \mathrm{pc}$ to $0.38 \mathrm{pc}$, molecular masses between $2.2 M_{\odot}$ and $22 M_{\odot}$, and virial masses from $70 M_{\odot}$ to $203 M_{\odot}$. All these clumps have a virial parameter $\alpha=M_{\mathrm{vir}} / M_{\mathrm{CO}} \gtrsim 6$ and are are consequently not gravitationally bound. It is unlikely that stars could have formed in these clumps. Clumps found in the SEST map have smaller masses, but show a similar picture, with $\alpha \gtrsim 5.5$.

Inside the molecular boundaries of MBM 18 we found ten candidate $\mathrm{H} \alpha$ emission-line stars from a low-resolution objective grism survey. To determine whether these $\mathrm{T}$ Tauri star 
Table 5. Parameters of the clumps identified in the KOSMA CO(1-0) data.

\begin{tabular}{|c|c|c|c|c|c|c|c|c|c|c|c|c|}
\hline Clump & $\begin{array}{l}\Delta \alpha \\
\quad(\operatorname{arc}\end{array}$ & ${ }_{\text {hin) }}^{\Delta \delta}$ & $\begin{array}{l}T_{\mathrm{mb}} \\
(\mathrm{K})\end{array}$ & $\begin{array}{l}V_{\mathrm{lsr}} \\
(\mathrm{km}\end{array}$ & $\begin{array}{l}\Delta V \\
-1)\end{array}$ & $\begin{array}{l}r_{1} \\
(\operatorname{ar}\end{array}$ & iin) & $r_{1}$ & $r_{2}$ & $\begin{array}{c}L_{\mathrm{CO}} \\
\mathrm{K} \mathrm{km} \mathrm{s}^{-1} \mathrm{pc}^{2}\end{array}$ & $M_{\mathrm{vir}}\left(r_{1}\right)$ & $M_{\mathrm{vir}}\left(r_{2}\right)$ \\
\hline 1 & -41.7 & -73.8 & 5.23 & 10.62 & 1.81 & 9.19 & 18.18 & 0.321 & 0.634 & 5.726 & 131.9 & 260.9 \\
\hline 2 & -14.6 & -54.9 & 4.69 & 10.85 & 1.84 & 6.61 & 14.25 & 0.231 & 0.497 & 2.490 & 98.9 & 213.0 \\
\hline 3 & -25.7 & -60.1 & 4.66 & 11.45 & 1.63 & 6.08 & 12.94 & 0.212 & 0.452 & 2.195 & 70.8 & 150.6 \\
\hline 4 & -51.1 & -41.3 & 4.65 & 10.42 & 1.64 & 8.72 & 16.72 & 0.304 & 0.583 & 3.337 & 102.7 & 196.7 \\
\hline 5 & -5.7 & 1.7 & 4.44 & 8.91 & 1.87 & 10.79 & 18.18 & 0.377 & 0.634 & 5.578 & 166.7 & 280.9 \\
\hline 6 & -33.4 & -49.0 & 3.11 & 10.62 & 1.46 & 8.44 & 13.32 & 0.295 & 0.465 & 1.655 & 79.4 & 125.4 \\
\hline 7 & -26.6 & 4.6 & 3.09 & 9.64 & 1.63 & 7.60 & 13.89 & 0.265 & 0.485 & 1.616 & 88.8 & 162.1 \\
\hline 8 & 1.2 & 34.7 & 2.57 & 9.16 & 2.31 & 8.67 & 12.54 & 0.303 & 0.438 & 1.582 & 203.2 & 293.7 \\
\hline 9 & 16.8 & 5.3 & 2.41 & 10.11 & 1.92 & 9.88 & 18.31 & 0.345 & 0.639 & 3.271 & 159.4 & 295.7 \\
\hline 10 & 6.7 & -32.0 & 2.31 & 11.13 & 1.62 & 6.04 & 10.55 & 0.211 & 0.368 & 0.969 & 69.7 & 121.6 \\
\hline 11 & -12.5 & 22.7 & 1.97 & 8.05 & 2.13 & 5.36 & 10.31 & 0.187 & 0.360 & 0.565 & 107.3 & 206.2 \\
\hline 12 & 0.8 & -21.0 & 1.95 & 9.61 & 1.35 & 9.15 & 12.74 & 0.319 & 0.445 & 0.727 & 73.7 & 102.5 \\
\hline
\end{tabular}
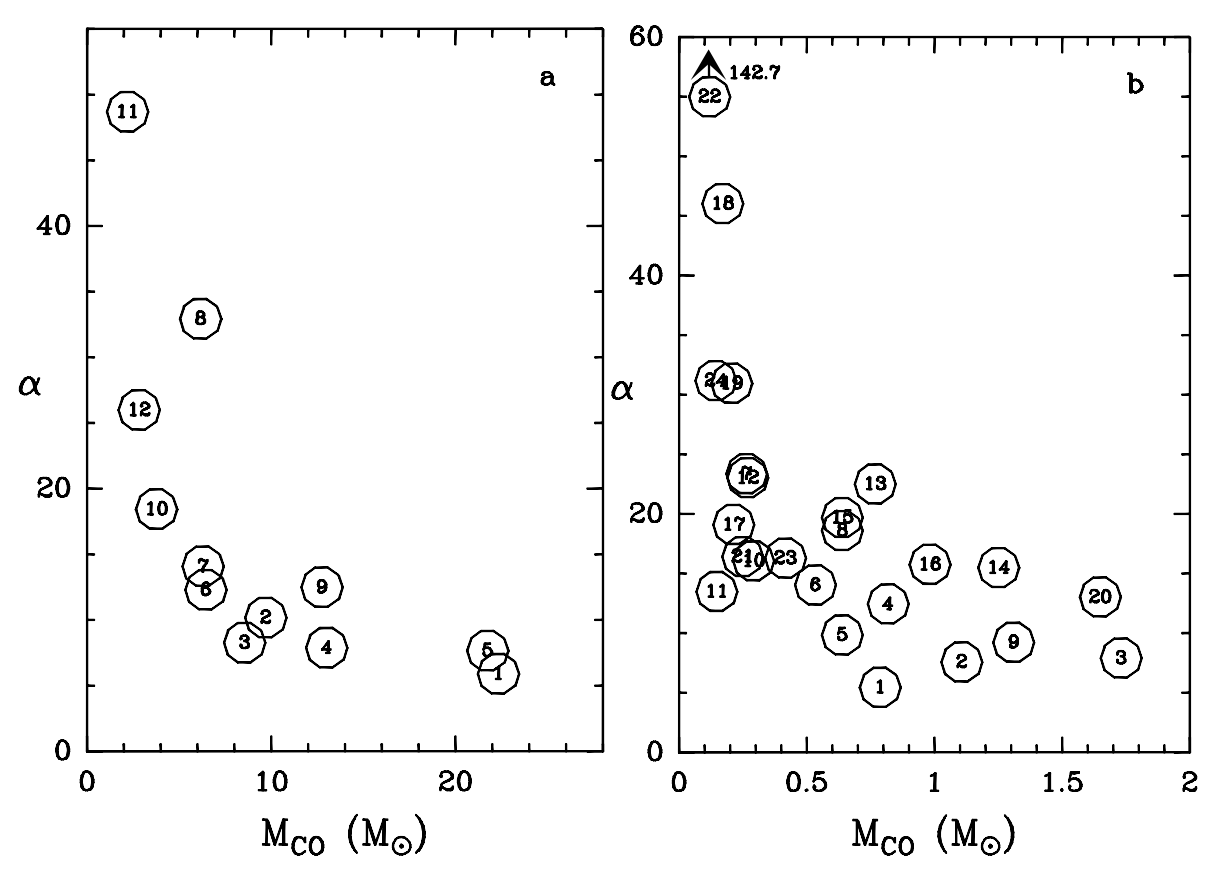

Fig. 14. a) Virial parameter $\alpha=M_{\mathrm{vir}}\left(r_{1}\right) / M_{\mathrm{CO}}$ as a function of $M_{\mathrm{CO}}=3.9 L_{\mathrm{CO}}$, for KOSMA-data. Data in Table 5. The numbers in the circles identify the clumps. In the absence of external pressure and magnetic fields, virial equilibrium is represented by $\alpha=1$. b) As a), but for SEST-data. Data in Table 6. For display purposes, clump 22 is not drawn at its real position, which is indicated with an arrow.

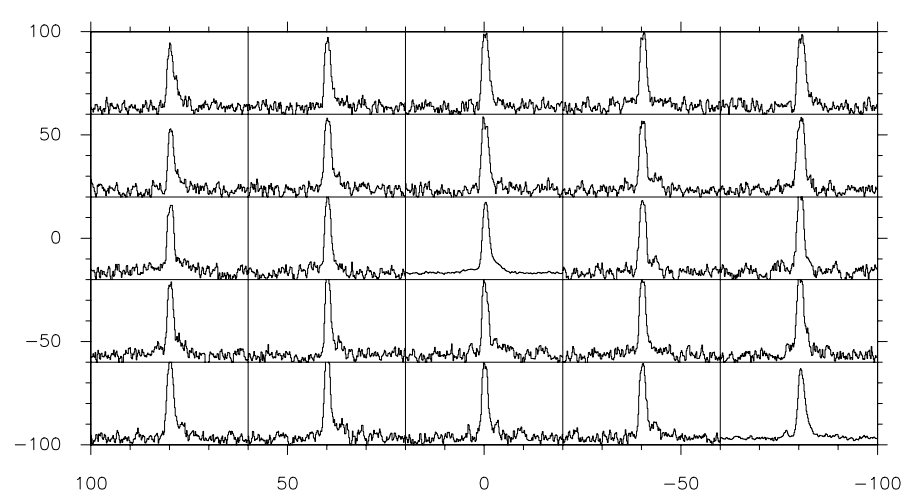

Fig. 15. Spectra of the ${ }^{12} \mathrm{CO}(1-0)$ emission in the central $200^{\prime \prime} \times 200^{\prime \prime}$ region of MBM 18 (SEST observations). Offsets are in arcseconds. For each spectrum the velocity scale runs from -5 to $+20 \mathrm{~km} \mathrm{~s}^{-1}$, and the temperature scale $\left(T_{\mathrm{A}}^{*}\right)$ from -0.5 to $5.5 \mathrm{~K}$. Note the non-Gaussian profiles at most positions.

candidates are indeed pre-main-sequence stars, we obtained higher-resolution spectra of seven of these stars to determine their spectral types, verify the presence of $\mathrm{H} \alpha$ emission and to

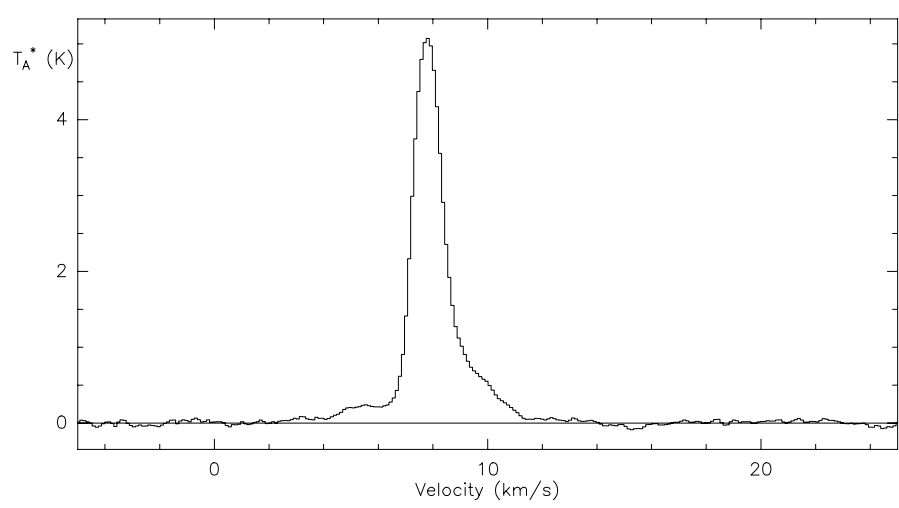

Fig. 16. Average ${ }^{12} \mathrm{CO}(1-0)$ spectrum of the central $400^{\prime \prime} \times 400^{\prime \prime}$ region of MBM 18 (SEST observations). The red and blue wings/pedestals are clearly visible.

try and detect the presence of LiI absorption. One of the observed stars $(\mathrm{Ha} 4)$ has the spectrum of a late F - early G-type star, with $\mathrm{H} \alpha$ in absorption. Its deviating position in the near- and mid-IR colour-colour diagrams may be caused by a companion 
Table 6. Parameters of the clumps identified in the SEST CO(1-0) data.

\begin{tabular}{cccccccccccccc}
\hline \hline Clump & $\begin{array}{c}\Delta \alpha \\
(\operatorname{arcmin})\end{array}$ & $\begin{array}{c}T_{\mathrm{mb}} \\
(\mathrm{K})\end{array}$ & \multicolumn{2}{c}{\begin{tabular}{c}
$V_{\mathrm{lsr}}\left(\mathrm{km} \mathrm{s}^{-1}\right)$ \\
\multicolumn{2}{c}{}
\end{tabular}} & $\begin{array}{c}r_{1} \\
(\mathrm{arcmin})\end{array}$ & $\begin{array}{c}r_{2} \\
(\mathrm{pc})\end{array}$ & $\begin{array}{c}r_{2} \\
\mathrm{~K} \mathrm{~km} \mathrm{~s}^{-1} \mathrm{pc}^{2}\end{array}$ & $\begin{array}{c}M_{\mathrm{vir}}\left(r_{1}\right) \\
\left(M_{\odot}\right)\end{array}$ \\
\hline 1 & -0.6 & -1.2 & 4.70 & 7.53 & 0.57 & 2.98 & 6.11 & 0.104 & 0.213 & 0.202 & 4.3 & 8.7 \\
2 & -18.9 & -6.7 & 4.56 & 9.49 & 0.73 & 3.61 & 7.63 & 0.126 & 0.266 & 0.284 & 8.4 & 17.7 \\
3 & -5.0 & 0.6 & 4.37 & 8.37 & 0.94 & 3.55 & 7.44 & 0.124 & 0.260 & 0.444 & 13.7 & 28.6 \\
4 & -2.9 & 12.7 & 4.27 & 8.29 & 0.94 & 2.64 & 4.99 & 0.092 & 0.174 & 0.210 & 10.2 & 19.2 \\
5 & 6.1 & 5.2 & 3.71 & 8.51 & 0.68 & 3.13 & 6.47 & 0.109 & 0.226 & 0.164 & 6.3 & 13.0 \\
6 & 0.7 & 7.4 & 3.53 & 8.00 & 0.83 & 2.46 & 4.87 & 0.086 & 0.170 & 0.137 & 7.5 & 14.8 \\
7 & -23.7 & -4.4 & 3.36 & 9.01 & 0.59 & 3.98 & 5.52 & 0.139 & 0.193 & 0.068 & 6.2 & 8.6 \\
8 & 5.1 & -1.9 & 3.42 & 8.37 & 0.99 & 2.79 & 5.58 & 0.097 & 0.195 & 0.164 & 11.9 & 23.8 \\
9 & -11.4 & -4.5 & 3.29 & 8.72 & 0.85 & 3.86 & 7.81 & 0.135 & 0.273 & 0.336 & 12.1 & 24.5 \\
10 & -25.3 & -12.2 & 3.51 & 9.17 & 0.69 & 2.27 & 4.45 & 0.079 & 0.155 & 0.075 & 4.7 & 9.2 \\
11 & -20.6 & -14.3 & 3.69 & 9.39 & 0.50 & 1.83 & 3.44 & 0.064 & 0.120 & 0.038 & 2.0 & 3.8 \\
12 & 14.3 & 9.6 & 3.56 & 8.64 & 0.85 & 1.93 & 3.83 & 0.067 & 0.134 & 0.069 & 6.2 & 12.2 \\
13 & -25.3 & 7.2 & 3.03 & 9.63 & 1.13 & 3.05 & 6.25 & 0.107 & 0.218 & 0.197 & 17.3 & 35.3 \\
14 & 18.3 & 3.8 & 2.76 & 10.70 & 0.95 & 4.89 & 9.54 & 0.171 & 0.333 & 0.321 & 19.4 & 37.8 \\
15 & 13.1 & -6.2 & 2.74 & 9.49 & 0.80 & 4.53 & 6.42 & 0.158 & 0.224 & 0.164 & 12.6 & 17.9 \\
16 & 25.7 & 6.1 & 2.73 & 10.18 & 0.90 & 4.39 & 7.92 & 0.153 & 0.277 & 0.252 & 15.5 & 28.0 \\
17 & -13.0 & -12.4 & 2.78 & 8.99 & 0.59 & 2.66 & 4.75 & 0.093 & 0.166 & 0.055 & 4.1 & 7.3 \\
18 & -6.8 & -9.6 & 2.73 & 7.41 & 0.90 & 2.22 & 3.83 & 0.078 & 0.134 & 0.044 & 7.9 & 13.7 \\
19 & -6.6 & 5.6 & 2.55 & 7.72 & 0.83 & 2.10 & 4.45 & 0.073 & 0.155 & 0.053 & 6.4 & 13.6 \\
20 & 8.5 & 9.0 & 2.18 & 9.49 & 0.95 & 5.48 & 10.37 & 0.191 & 0.362 & 0.423 & 21.5 & 40.7 \\
21 & -32.1 & 3.6 & 2.26 & 8.80 & 0.49 & 3.94 & 5.63 & 0.138 & 0.196 & 0.064 & 4.1 & 5.9 \\
22 & -9.5 & 10.8 & 2.27 & 8.21 & 1.21 & 2.61 & 3.27 & 0.091 & 0.114 & 0.030 & 16.7 & 21.0 \\
23 & 15.0 & 12.6 & 2.20 & 8.92 & 0.67 & 3.39 & 6.06 & 0.118 & 0.212 & 0.107 & 6.8 & 12.1 \\
24 & -31.0 & -8.1 & 2.30 & 9.19 & 0.66 & 2.38 & 3.76 & 0.083 & 0.131 & 0.037 & 4.5 & 7.2 \\
\hline
\end{tabular}

that is affecting the colours, but remains undetected in the spectrum. The other six stars are M-type dwarfs of type M1.5-M4.0 (see Table 4). Only four out of six have $\mathrm{H} \alpha$ emission, none has other emission lines, and no strong (or indeed any) LiI absorption (only three stars show a mere hint) has been detected, implying that they are not T Tauri stars. From the observed colours and the intrinsic colours determined from the spectral types, we derive distances to the six M-stars of between 60 and $250 \mathrm{pc}$. We did obtain a good fit of the late-type stars on pre-main-sequence isochrones, finding ages between 7.5 and 15 Myr. By this time any lithium is likely to have been depleted. Only three of our target stars (viz Ha1, 2, and 8) lie near or within the generally accepted range of distances (120-150 pc) of MBM 18. If these stars were indeed formed in the cloud, MBM 18 would be considerably older than a typical translucent cloud. Considering that only unbound clumps are found, a formation in situ is unlikely.

Acknowledgements. This work is partly based on observations made with the Italian Telescopio Nazionale Galileo (TNG) operated on the island of La Palma by the Fundación Galileo Galilei of the INAF (Istituto Nazionale di Astrofisica) at the Spanish Observatorio del Roque de los Muchachos of the Instituto de Astrofisica de Canarias (programmes TAC34-AOT19 and TAC17-AOT22). The TNG-observations were carried out in service mode. We are grateful to our support astronomers Vania Lorenzi (2009 observations) and Francesca Ghinassi (2010 observations) for their assistance. We thank Giovanna Stirpe for help with the TNG-data reduction. At the time of the observations, the KOSMA radio telescope at Gornergrat-Süd Observatory was operated by the University of Köln, and supported by the Deutsche Forschungsgemeinschaft through grant SFB-301, as well as by special funding from the Land Nordrhein-Westfalen. The observatory was administered by the Internationale Stiftung Hochalpine Forschungsstationen Jungfraujoch und Gornergrat, Bern, Switzerland. The telescope is currently located in Tibet. This research has made use of the SIMBAD database, operated at CDS, Strasbourg, France, and of NASA's Astrophysics Data System Bibliographic Services (ADS). This publication makes use of data products from the Wide-field Infrared Survey Explorer, which is a joint project of the University of California, Los Angeles, and the Jet Propulsion Laboratory/California Institute of Technology, funded by the National Aeronautics and Space Administration. We made use of observations with the 100-m telescope of the Max-Planck-Institut für Radioastronomie at Effelsberg. We thank Adam Schneider for help in producing Fig. 12, and Steven Shore for comments on an earlier version of the paper.

\section{References}

Appenzeller, I., \& Mundt, R. 1989, A\&ARv, 1, 291

Bachiller, R. 1996, ARA\&A, 34, 111

Barriault, L., Joncas, G., Falgarone, E., et al. 2010, MNRAS, 406, 2713 Bertout, C. 1989, ARA\&A, 27, 351

Bessel, M. S., \& Brett, J. M. 1988, PASP, 100, 1134

Blitz, L., Magnani, L., \& Wandel, A. 1988, ApJ, 331, 127

Bohlin, R. C., Savage, B. D., \& Drake, J. F. 1978, ApJ, 224, 132

Carpenter, J. M. 2001, AJ, 121, 2851

Chaplin, V. H., Larson, K. A., \& Gerakines, P. A. 2004, BAAS, 36, 1439 Cox, A. N. 2000, Allen's Astrophysical Quantities, 4th edn. (Springer) Cruz, K. L., \& Reid, I. N. 2002, AJ, 123, 2828

Dame, T. M., Hartmann, D., \& Thaddeus, P. 2001, ApJ, 547, 792 de la Reza, R., Torres, C. A. O., Quast, G., et al. 1989, ApJ, 343, L61 Dickman, R. L., \& Clemens, D. P. 1983, ApJ, 271, 143

Falgarone, E., \& Phillips, T. G. 1990, ApJ, 359, 344

Felli, M., Brand, J., Cesaroni, R., et al. 2007, A\&A, 476, 373

Franco, G. A. P. 1988, A\&A, 202, 173

Hearty, T. J. III 1997, Ph.D. Thesis, University of Georgia

Harju, J., Walmsley, C. M., \& Wouterloot, J. G. A. 1993, A\&AS, 98, 51

Jacoby, G. H., Hunter, D. A., \& Christian, C. A. 1984, ApJS, 56, 257 Joy, A. H., \& Abt, H. A. 1974, ApJS 28, 1

Luhman, K. L. 2001, ApJ, 560, 287

Magnani, L. 1988, in The Outer Galaxy, Lect. Notes Phys., eds. L. Blitz, \& F. J. Lockman (Springer-Verlag), 306, 168

Magnani, L., \& de Vries, C. P. 1986, A\&A, 168, 271

Magnani, L., Blitz, L., \& Mundy, L. 1985, ApJ, 295, 402 (MBM) Magnani, L., Carpenter, J. M., Blitz, L., et al. 1990, ApJS, 73, 747

Magnani, L., Caillault, J.-P., Hearty, T., et al. 1996a, ApJ, 465, 825 Magnani, L., Hartmann D., \& Speck B. G. 1996b, ApJS, 106, 447 Magnani, L., Hartmann, D., Holcomb, S. L., et al. 2000, ApJ, 535, 167 McGehee, P. 2008, in Handbook of Star Forming Regions, ed. B. Reipurth, Publ. Astron. Soc. Pacific, MP005, 2, 813

Neuhäuser, R., Sterzik, M. F., Schmitt, J. H. M. M., et al. 1995, A\&A, 295, L5 Osterbrock, D. E., Fulbright, J. P., Martel, A. R., et al. 1996, PASP, 108, 277 Ott, M., Witzel, A., \& Quirrenbach, A. 1994, A\&A, 284, 331

Palla, F., \& Stahler, S. W. 1999, ApJ, 525, 772

Penprase, B. E. 1992, ApJS, 83, 273

Penprase, B. E. 1993, ApJS, 88, 433

Penprase, B. E., Blades, J. C., Danks, A. C., \& Crane, P. 1990, ApJ, 365, 241

Pound, M. W., Wilson, R. W., \& Bania, T. M. 1990, ApJ, 351, 165

Preibisch, T., Guenther, E., Zinnecker, H., et al. 1998, A\&A, 333, 619

Rieke, G. H., \& Lebofsky, M. J. 1985, ApJ, 288, 618 
A\&A 547, A85 (2012)

ROSAT 2000, ROSAT News, 72

Schlegel, D. J., Finkbeiner, D. P., \& Davis, M. 1998, ApJ, 500, 525

Schmidt-Kaler, Th. 1982, in Landolt-Börnstein, VI/2b (Springer)

Shore, S. N., Magnani, L., LaRosa, T. N., \& McCarthy, M. N. 2003, ApJ, 593, 413

Shore, S. N., LaRosa, T. N., Chastain, R. J., \& Magnani, L. 2006, A\&A, 457 197

Stahler, S. W., \& Palla, F. 2004, The Formation of Stars (Wiley-VCH)

Turnshek, D. E., Turnshek, D. A., Craine, E. R., \& Boeshaar, P. C. 1985, An Atlas of Digital Spectra of Cool Stars (Tucson: Western Research Company)
White, N. E., Giommi, P., \& Angelini, L. 2000, VizieR on-line data catalogue IX/31 (Strassbourg: SIMBAD)

Whitworth, A. P., Bhattal, A. S., Chapman, S. J., et al. 1994, MNRAS, 268, 291

Williams, J. P., de Geus, E. J., \& Blitz, L. 1994, ApJ, 428, 693

Wouterloot, J. G. A., \& Brand, J. 1992, A\&A, 265, 144

Wright, E. L., Eisenhardt, P. R. M., Mainzer, A. K., et al. 2010, AJ, 140, 1868

Zheng, Z., Flynn, C., Gould, A., et al. 2001, ApJ, 555, 393

Zuckerman, B., Webb, R. A., Schwartz, M., \& Becklin, E. E. 2001, ApJ, 549, L233

Pages 17 to 20 are available in the electronic edition of the journal at http://www . aanda. org 


\section{Appendix A: Spectra of the candidate $\mathrm{H} \alpha$ emission-line stars}

In Fig. A1 we present the full spectra of the M-dwarf stars in our sample. In Fig. A2 we show the spectrum of the earlier type star.
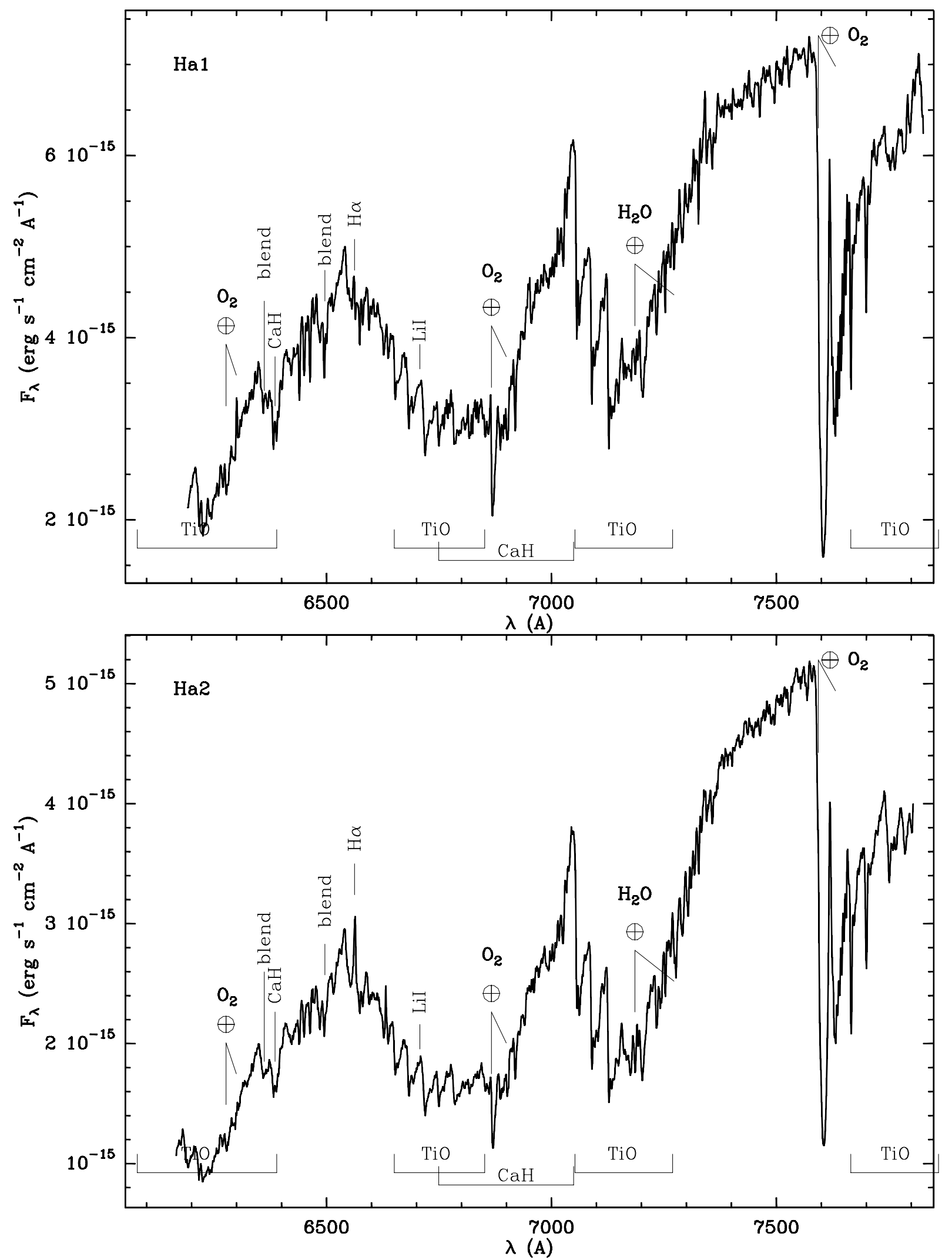

Fig. A.1. a) (Top panel) Spectrum of candidate $\mathrm{H} \alpha$ emission-line star $\mathrm{Ha} 1$. The locations of the $\mathrm{TiO}$ and $\mathrm{CaH}$ absorption bands are indicated below the spectrum, as are the expected locations of several features. Telluric lines are marked with a crossed circle. b) (Bottom panel) as a), but for candidate $\mathrm{H} \alpha$ emission-line star $\mathrm{Ha} 2$. 

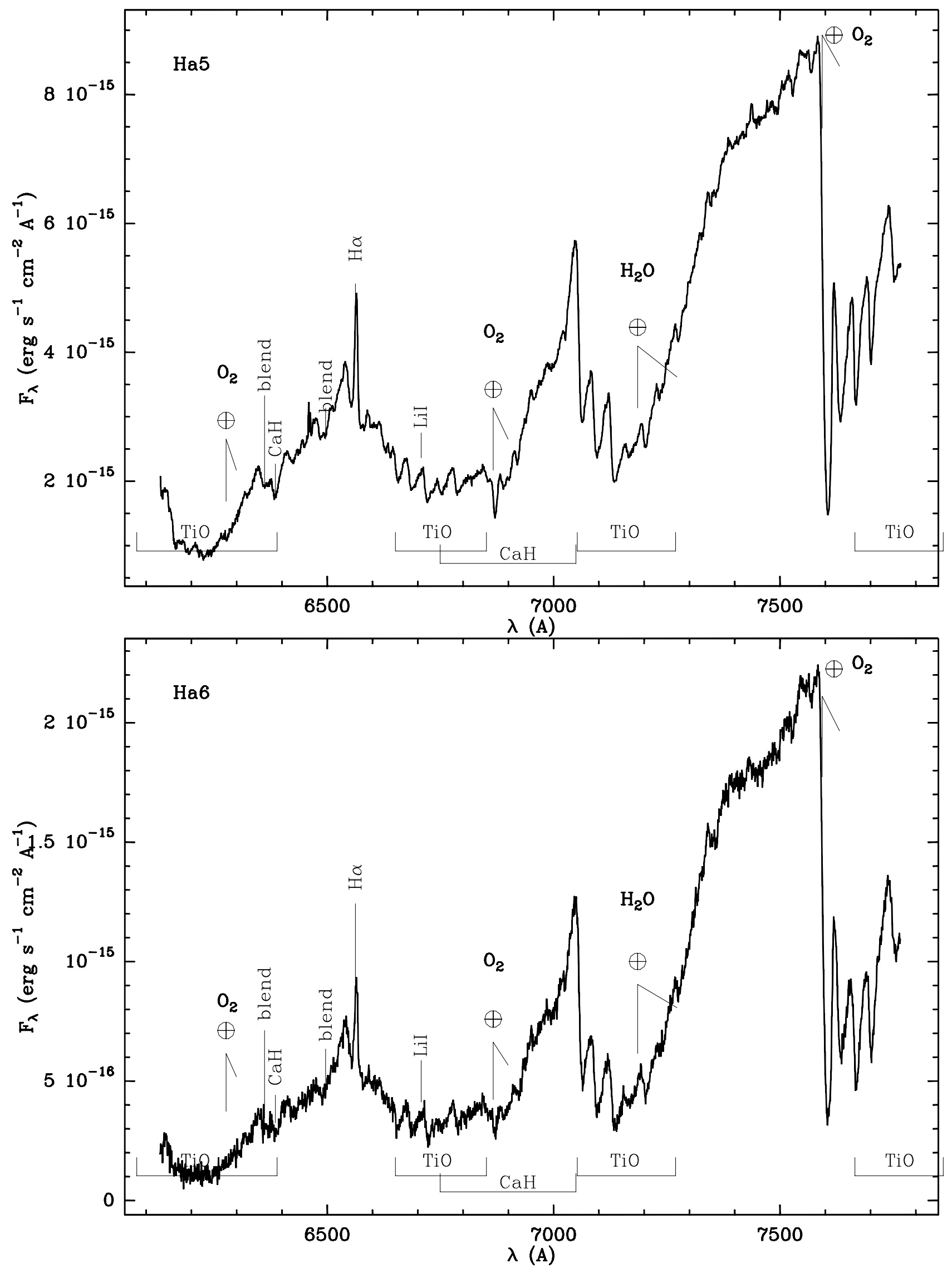

Fig. A.1. c) (Top panel) As a), for candidate H $\alpha$ emission-line star Ha5. d) (Bottom panel) as c), but for candidate H $\alpha$ emission-line star Ha6. 
J. Brand et al.: Molecular gas and stars in the translucent cloud MBM 18 (LDN 1569)
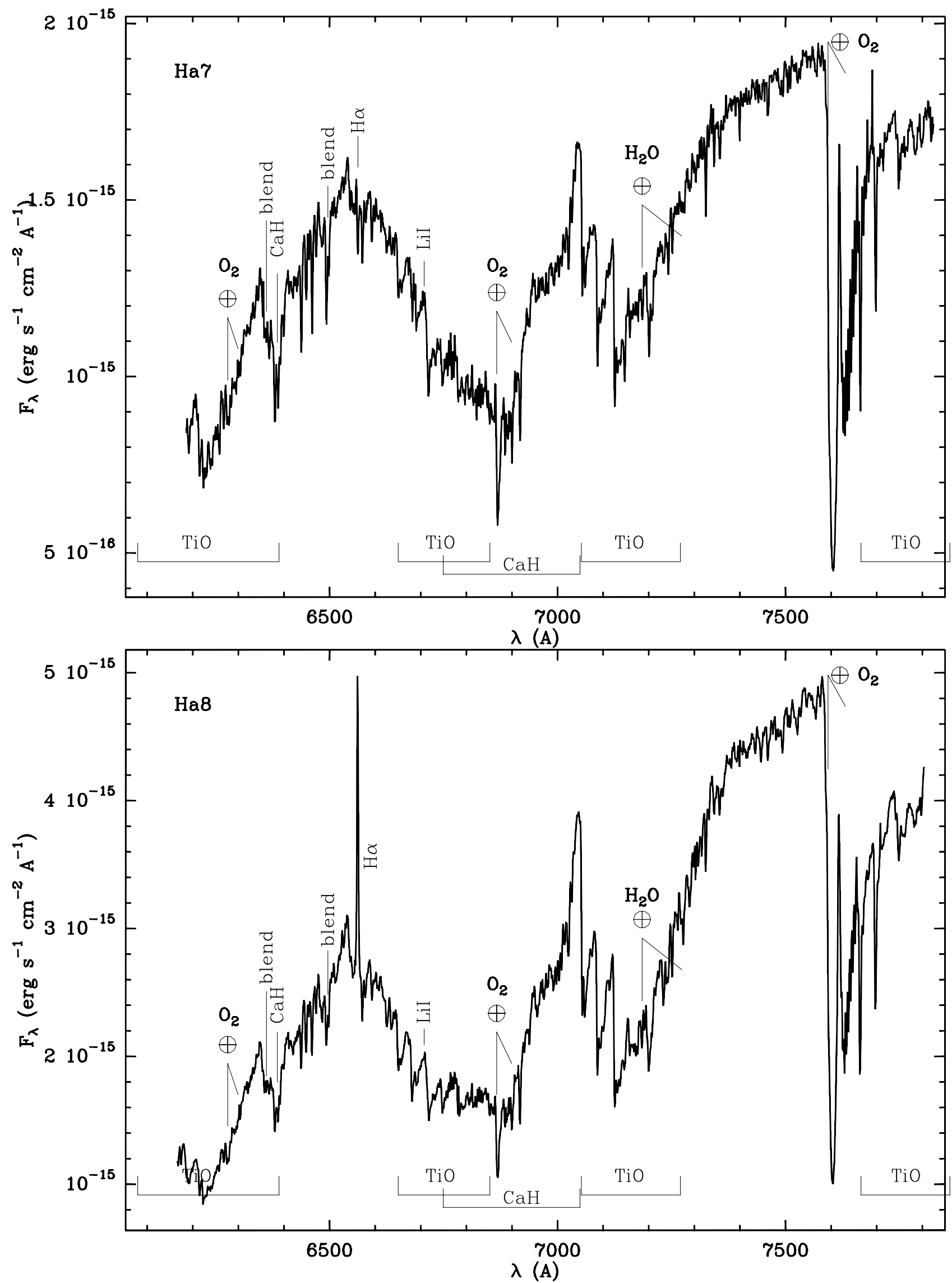

Fig. A.1. e) (Top panel) As a), for candidate $\mathrm{H} \alpha$ emission-line star Ha7. f) (Bottom panel) as e), but for candidate $\mathrm{H} \alpha$ emission-line star Ha8. 
A\&A 547, A85 (2012)

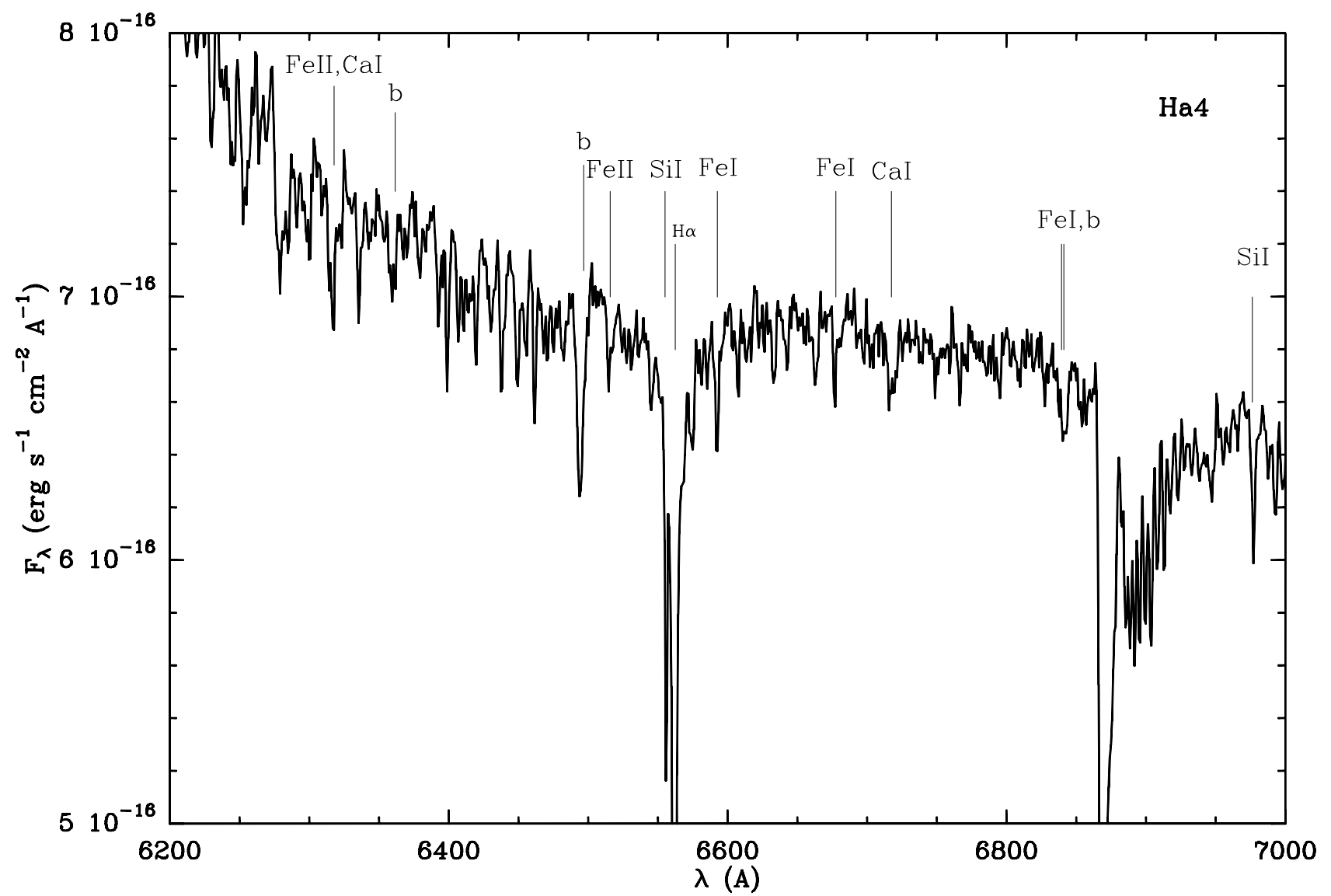

Fig. A.2. The spectrum of candidate $\mathrm{H} \alpha$ emission-line star Ha4. Several absorption lines are indicated. This star is clearly of earlier spectral type than the others. 